\title{
INFINITE PARTICLE SYSTEMS
}

\author{
BY
}

\author{
SIDNEY C. PORT AND CHARLES J. STONE
}

\begin{abstract}
We consider a system of denumerably many particles that are distributed at random according to a stationary distribution $P$ on some closed subgroup $X$ of Euclidean space. We assume that the expected number of particles in any compact set is finite. We investigate the relationship between $P$ and the distribution $Q$ of-particles as viewed from a particle selected "at random" from some set. The distribution $Q$ is called the tagged particle distribution. We give formulas for computing $P$ in terms of $Q$ and $Q$ in terms of $P$ and show that, with the appropriate notion of convergences, $P_{n}$ $\rightarrow P$ implies $Q_{n} \rightarrow Q$ and vice versa. The particles are allowed to move in an appropriate translation invariant manner and we show that the tagged particle distribution $Q^{\prime}$ at a later time 1 is the same as the distribution of particles at time 1 as viewed from a particle selected "at random" from those initially in some set. We also show that $Q^{\prime}$ is the same as the distribution of particles at time 1 as viewed from a particle selected at random from those at the origin, when initially the particles are distributed according to $Q$. The one-dimensional case is treated in more detail. With appropriate topologies, we show that in this case there is a homeomorphism between the collection of stationary distributions $P$ and tagged particle distributions $Q$. A stationary spacings distribution $Q_{0}$ related to $Q$ is introduced, and we show that with the appropriate topology the map taking $Q$ to $Q_{0}$ is a homeomorphism. Explicit expressions are found for all these maps and their inverses. The paper concludes by using the one-dimensional results to find stationary distributions for a class of motions of denumerably many unit intervals and to establish criteria for convergence to one of these distributions.
\end{abstract}

1. Introduction. In this paper we investigate several topics involving infinite particle systems in Euclidean space. The work was suggested especially by the papers of Ryll-Nardzewski [5], Slivnyak [6], Mecke [4], Harris [2] and [3], Spitzer [7], and Stone [8]. Other related work can be found in the references in [3]. In $\$ \$ 2-7$ essentially what we do is extend to the general case results that had previously been known under one or more of the following restrictions: no multiple occupancy; one-dimension; no marks. Of these restrictions the first is the most serious and getting rid of it usually first requires a proper reformulation of the results. In $\$ 8$ we apply our results to study the motion of a system of "hard rods." This application actually motivated much of the present work.

Received by the editors February 22, 1972 and, in revised form, April 24, 1972. AMS (MOS) subject classifications (1970)。 Primary 60G99; Secondary 60K35.

Key words and phrases. Infinite particle system, random counting measure, tagged particle distribution, point process.

(1) The preparation of this paper was supported in part by NSF Grant GP-28258。 
Let $X$ be a closed subgroup of $R^{d}$. We consider a system of denumerably many particles in $X$. Associated with each particle is a mark $m$, where $m$ lies in a complete separable metric space $M$. Typical marks are the momentum or velocity of the particle. We actually do not use the marks in any significant way, but they can be included with very little effort and may be useful in future applications. At any rate, the case of no marks is covered by letting $M$ be a set consisting of a single point.

We assume that there are only a finite number of particles in any compact subset of $X$. By ordering the particles we are led to a sequence $\left\{\left(x_{i}, m_{i}\right)\right\}$ of elements of $X \times M$. The equation

$$
\iint \omega(d x, d m) f(x, m)=\sum_{i} f\left(x_{i}, m_{i}\right)
$$

defines a counting measure $\omega$ on $X \times M$ such that $\omega(A \times M)<\infty$ for every compact subset $A$ of $X$. From now on, by $\left\{\left(x_{i}, m_{i}\right)\right\}$ we really mean the counting measure $\omega$ defined by (1.1).

The set $\Omega$ of such counting measures can be made into a complete separable metric space. By a measurable subset of $\Omega$ we mean a Borel set. By introducing the appropriate probability structure, we can think of $\left\{\left(x_{i}, m_{i}\right)\right\}$ as a random process, called a marked process on $X$. The process is said to be stationary if $\left\{\left(x_{i}+t, m_{i}\right)\right\}$ has the same distribution as $\left\{\left(x_{i}, m_{i}\right)\right\}$ for all $t \in X$.

Consider a stationary marked process having distribution $P$. If the expected number of particles in some nonempty open set is finite, there is a constant $a(P)$ such that the expected number of particles in the set $A$ having Haar measure $|A|$ is just $\alpha(P)|A|$. Otherwise we set $\alpha(P)=\infty$.

Consider a stationary marked process having distribution $P$ such that $0<$ $a(P)<\infty$. In $\$ 3$ we investigate the relationship between $P$ and the distribution $Q$ of particles viewed from the position of a particle selected "at random" from some set $A$. In order to give this a precise formulation, we let $\omega+t$ correspond to $\left\{\left(x_{i}+t, m_{i}\right)\right\}$ as $\omega$ corresponds to $\left\{\left(x_{i}, m_{i}\right)\right\}$. We show that there is a unique probability distribution $Q$ on $\Omega$ such that for any Borel set $A$ in $X$ with $|A|<\infty$ and any bounded measurable function $f$ on $\Omega$

$$
\alpha(P)|A| \int Q(d \omega) f(\omega)=\int P(d \omega) \sum_{x_{i} \in A} f\left(\omega-x_{i}\right) .
$$

The measure $Q$, which assigns probability one to $\{\omega: \omega(\{0\} \times M)>0\}$, is called the tagged particle distribution corresponding to $P$. Theorem 3.2 is essentially contained in Theorem 2.3 of Mecke [4].

As an example, in $\$ 3$ we let $P$ be a compound Poisson process in which the 
number of particles at a site where particles are present has distribution $F$. Then $Q$ differs from $P$ only in that, independently of the behavior of the process away from the origin, a random number of particles is placed at the origin, whose distribution $G$ is given by

$$
G(d x)=x F(d x) / \int_{0}^{\infty} x F(d x)
$$

In $\S 4$ we use the multidimensional ergodic theorem to verify the existence of certain limits involving $\omega$. In terms of these limits we give a formula for computing $P$ in terms of $Q$ and $\alpha(P)$.

Let $P_{n}, n \geq 1$, and $P$ be distributions of stationary marked processes such that $\alpha\left(P_{n}\right) \rightarrow \alpha(P)$ as $n \rightarrow \infty, \alpha\left(P_{n}\right)$ and $\alpha(P)$ being assumed finite and positive. Let $Q_{n}, n \geq 1$, and $Q$ be the corresponding tagged particle distributions. In $\$ 5$ we show that $P_{n} \rightarrow P$ weakly if and only if $Q_{n} \rightarrow Q$ weakly. The present version of the first part of the proof of Theorem 5.1 was suggested by the referee, whose suggestions also led to simplifications in the derivations in $\$ 4$.

Suppose that initially at time 0 the particles are distributed according to $\left\{\left(x_{i}, m_{i}\right)\right\}$ and that at some later time, say time 1 , the $i$ th particle is at $x_{i}^{\prime}$ and has the mark $m_{i}^{\prime}$. The process $\left\{\left(x_{i}, m_{i}, x_{i}^{\prime}, m_{i}^{\prime}\right)\right\}$ is called a marked motion process and may be precisely defined by means of an equation similar to (1.1) as a random counting measure on $X \times M \times X \times M$. The marked process is said to be translation invariant if $\left\{\left(x_{i}+t, m_{i}, x_{i}^{\prime}+t, m_{i}^{\prime}\right)\right\}$ has the same distribution as $\left\{\left(x_{i}, m_{i}, x_{i}^{\prime}, m_{i}^{\prime}\right)\right\}$ for all $t \in X$. Let $P$ and $P^{\prime}$ denote the distributions of $\left\{\left(x_{i}, m_{i}\right)\right\}$ and $\left\{\left(x_{i}^{\prime}, m_{i}^{\prime}\right)\right\}$. If the marked motion process is translation invariant, then $P$ and $P^{\prime}$ are stationary and $\alpha(P)=\alpha\left(P^{\prime}\right)$.

Suppose we are given a translation invariant marked motion process such that $0<a(P)=a\left(P^{\prime}\right)<\infty$. Let $Q$ and $Q^{\prime}$ denote the tagged particle distributions corresponding to $P$ and $P^{\prime}$. In $\$ 6$ we show that $Q^{\prime}$ is the distribution of the marked process at time 1 viewed from the position at time 1 of a particle selected "at random" from those initially in some fixed set. We also show that $Q^{\prime}$ is the distribution of the marked point process at time 1 viewed from the position at time 1 of a particle selected at random from among those particles initially at the origin, when the particles initially are distributed according to $Q$. (To do this, of course, we need to define a marked motion process corresponding to an arbitrary initial distribution.) If the distribution $P$ is such that with probability one no two particles occupy the same location, then the process distributed according to $Q$ has exactly one particle at the origin. In this case $Q^{\prime}$ is the distribution of the marked process at time 1 viewed from the position at time 1 of the particle initially at the origin, when the initial distribution is $Q$. The results of this section were strongly suggested by similar results in $\$ 6$ of Harris [3] under the 
restriction of no multiple occupancy and in Spitzer [7, pp. 272 and 280], where some particular models allowing multiple occupancy are considered which involve motion of particles on a countable set.

In $\$ 7$ we consider the one-dimensional case in more detail. Let $\mathcal{P}$ denote the collection of all distributions $P$ of stationary marked processes such that $\alpha(P)$ $<\infty$ and $P(\omega=0)=0$. Let 2 denote the collection of the corresponding tagged particle distributions. We determine 2 explicitly. We show that, in terms of the appropriate topologies, the map that takes $P$ into the corresponding $Q$ is a homeomorphism of $\mathcal{P}$ onto 2 . We find closed form expressions for this map and its inverse. Let $2_{0}$ denote the collection of distributions of stationary sequences $\left\{\left(\eta_{i}, m_{i}\right)\right\}$ such that $\eta_{i} \geq 0, \eta_{0}$ has finite expectation, and $\Sigma_{-\infty}^{-1} \eta_{i}=\Sigma_{0}^{\infty} \eta_{i}=\infty$ with probability one. We show that if $2_{0}$ is suitably topologized there is a natural homeomorphism between 2 and $2_{0}$ which, together with its inverse, can be written in closed form. As a consequence we also get closed formed expressions for the composed map from $\mathcal{P}$ to $\mathscr{Q}_{0}$ and its inverse. The distribution $Q_{0} \in \mathscr{Q}_{0}$ corresponding to $P \in \mathcal{P}$ is called the spacing distribution corresponding to $P$.

The results of $\$ 7$ are applied in $\$ 8$ to study the behavior of the motion of a system of "rods." These rods, by definition, are unit intervals on the real line which are constrained not to have any interior points in common at any time. We consider a fairly general family of motions that satisfy this constraint. In the simplest setting, when these rods move with random velocities, two rods exchange velocities when they collide. We show that the system of moving rods has a stationary distribution $P$ which is a stationary renewal process, so that the corresponding spacing process $\left\{\eta_{i}\right\}$ is a sequence of independent identically distributed random variables; moreover $\eta_{i}-1$ is exponentially distributed. We also give conditions which guarantee convergence to this stationary distribution as time approaches infinity. The basic method used is to apply the results of $\$ 7$ to reduce the results for the interacting motion of the rods to the corresponding results about independent motion of particles.

2. Preliminaries. Let $X$ be a $d$-dimensional closed subgroup of $R^{d}$, which we can assume to be of the form

$$
X=\left\{x=\left(x^{1}, \ldots, x^{d}\right): x^{k} \in Z \text { for } 1 \leq k \leq d_{1}\right\},
$$

where $Z$ denotes the integers. The most interesting cases are $X=R^{d}$ when $d_{1}=0$ and $X=Z^{d}$ when $d_{1}=d$. We let || denote Haar measure on $X$, defined as the product of counting measure on the first $d_{1}$ coordinates of $X$ and Lebesgue measure on the last $d-d_{1}$ coordinates of $X$.

Let $M$ denote a complete separable metric space. By measurable sets in $M$ we mean the Borel sets. 
A marked counting measure $\omega$ on $X \times M$ is an integer-valued measure on $X \times M$ such that $\omega(A \times M)<\infty$ for each compact subset $A$ of $X$. Associated with each measure $\omega$ is its realization as a sequence of points $\left\{\left(x_{i}, m_{i}\right)\right\}$ where

$$
\iint \omega(d x, d m) f(x, m)=\sum_{i} f\left(x_{i}, m_{i}\right) .
$$

Let $\Omega$ be the collection of all marked counting measures on $X \times M$. We can make $\Omega$ into a complete separable metric space in such a manner that $\omega_{n} \rightarrow \omega$ as $n \rightarrow \infty$ if and only if

$$
\lim _{n \rightarrow \infty} \iint \omega_{n}(d x, d m) f(x, m)=\iint \omega(d x, d m) f(x, m)
$$

for all bounded continuous functions $f$ having support in a set of the form $A \times M$ where $A$ is compact. By a measurable set in $\Omega$ we mean a Borel set. By a marked process, we mean a random marked counting measure distributed according to some probability measure $P$ on $\Omega$.

For $\omega \in \Omega$ and $t \in X$, we define $\omega+t$ to be the marked counting measure given by

$$
\iint(\omega+t)(d x, d m) f(x, m)=\iint \omega(d x, d m) f(x+t, m)
$$

Thus $(\omega+t)(A \times M)=\omega(A-t, M)$. Observe that $\omega+t$ is a jointly continuous function of $\omega$ and $t$.

Consider a marked process having distribution $P$. The measure $P$ and the process are called stationary if

$$
\int P(d \omega) f(\omega+t)=\int P(d \omega) f(\omega)
$$

for all $t \in X$ and bounded measurable functions $f$ on $\Omega$.

It is convenient to introduce the notation $N(A)=N(A, \omega)=\omega(A \times M)$ for Borel subsets $A$ of $X$ and $N(x)=N(\{x\})$ for $x \in X$.

Suppose $P$ is a stationary distribution such that $E N(A)=\int P(d \omega) N(A)<\infty$ for some nonempty open subset $A$ of $X$. By stationarity, it follows that $E N(A)$ $<\infty$ for all compact subsets $A$ of $X$. Set $\mu(A)=E N(A)$ for all Borel subsets $A$ of $X$. Then $\mu$ is a Radon measure on $X$ such that, for all Borel sets $A \subset X$ and all $t \in X, \mu(A+t)=\mu(A)$. By the uniqueness of Haar measure there is a nonnegative constant $a(P)$ such that $\mu(A)=\alpha(P)|A|$, where $|A|$ is the Haar measure of $A$. Thus for all Borel sets $A \subset X, E N(A)=\alpha(P)|A|$. If $E N(A)=\infty$ for every nonempty open set $A \subset X$, we set $\alpha(P)=\infty$. 
Let $\Omega^{*}$ denote the set of $\omega \in \Omega$ such that $N(B)=\infty$ for each of the $2^{d}$ orthants of $X$. Observe that $\Omega^{*}$ is not complete.

Theorem 2.1. Let $P$ be the distribution of a stationary marked process. Then

$$
P\left(\Omega^{*}\right)=P(N(X)>0) \text {. }
$$

Proof. Let $B=\left\{x: 0 \leq x^{i}<\infty, 1 \leq i \leq d\right\}$ denote the positive orthant of $X$ and set $u=(1, \ldots, 1) \in B$. Since $B-n u \uparrow X$ and $B+n u \downarrow \varnothing$ as $n \rightarrow \infty$, it follows that $\lim _{n \rightarrow \infty} N(B-n u)=N(X)$ and $\lim _{n \rightarrow \infty} N(B+n u)=0$ if $N(B)<\infty$. Thus

$$
\lim _{n \rightarrow \infty} P(N(B-n u)=0)=P(N(X)=0)
$$

and

$$
\lim _{n \rightarrow \infty} P(N(B+n u)=0) \geq P(N(B)<\infty)
$$

By stationarity, $P(N(B \pm n u)=0)=P(N(B)=0)$ so (2.3) implies that $P(N(B)=0)$ $\geq P(N(B)<\infty)$ and hence that

$$
P(N(B)=0)=P(N(B)<\infty) .
$$

We conclude from (2.2) and (2.4) that

$$
P(N(B)<\infty)=P(N(X)=0) .
$$

Similarly (2.5) holds for each of the other $2^{d}-1$ orthants of $X$ and hence (2.1) holds.

For a Borel set $A \subset X$, let $N^{*}(A)=N^{*}(A, \omega)$ denote the number of distinct sites in $A$ occupied by particles. By definition

$$
N^{*}(A)=\sum_{i} 1_{A}\left(x_{i}\right) / N\left(x_{i}\right)
$$

Clearly $0 \leq N^{*}(A) \leq N(A)$ and $N^{*}(A)>0$ if and only if $N(A)>0$.

Let $\omega$ be distributed according to a stationary distribution $P$ such that $\alpha(P)<\infty$. Then there is a nonnegative constant $\lambda(P)$ such that $E N^{*}(A)=$ $\lambda(P)|A|$ for all Borel sets $A \subset X$ having finite measure. Clearly $0 \leq \lambda(P) \leq a(P)$ and $\lambda(P)>0$ if and only if $\alpha(P)>0$. Since $E N(A)=\alpha(P)|A|$, we conclude that if $0<|A|<\infty$, then $N(A)=N^{*}(A)$ with probability one if and only if $\alpha(P)=\lambda(P)$. Thus $\alpha(P)=\lambda(P)$ if and only if $N=N^{*}$ with probability one or, equivalently, if and only if, with probability one, $\omega$ assigns distinct locations to each of the particles.

For $r \geq 0$, set $\Delta_{r}=\left\{x \in X:\left|x^{i}\right| \leq r, i=1,2, \ldots, d\right\}$. The next result extends Korolyook's theorem. 
Theorem 2.2. Consider a stationary marked process baving distribution $P$ such that $a(P)<\infty$. Then

$$
\alpha(P)=\lim \frac{1}{\left|\Delta_{r}\right|} E\left[N\left(\Delta_{r}\right) 1_{\left\{N^{*}\left(\Delta_{r}\right)=1\right\}}\right] .
$$

Proof. For $r>0$ set $\Gamma_{r}=\left\{x \in X: 0 \leq x^{i}<r, i=1,2, \ldots, d\right\}$. To prove (2.6) it suffices to show that

$$
\alpha(P)=\lim _{r \rightarrow 0} \frac{1}{\left|\Gamma_{r}\right|} E N\left(\Gamma_{r}\right) 1_{\left\{N^{*}\left(\boldsymbol{\Gamma}_{r}\right)=1\right\}}
$$

To verify (2.7) we let $N_{r, n}\left(\Gamma_{1}\right), n \in Z^{d}$, be the random variable defined by $\left.N_{r, n}\left(\Gamma_{1}\right)=N\left(n r+\Gamma_{r}\right) 1 N^{*}\left(n r+\Gamma_{r}\right)=1\right\}$ if $n r+\Gamma_{r} \subset \Gamma_{1}$ and $N_{r, n}\left(\Gamma_{1}\right)=0$ otherwise. We also set $N_{r}\left(\Gamma_{1}\right)=\sum_{n} N_{r, n}\left(\Gamma_{1}\right)$. Then $0 \leq N_{r}\left(\Gamma_{1}\right) \leq N\left(\Gamma_{1}\right)$ and, with probability one, $N_{r}\left(\Gamma_{1}\right)=N\left(\Gamma_{1}\right)$ for $r$ sufficiently small. Thus by dominated convergence

$$
\alpha(P)=E N\left(\Gamma_{1}\right)=\lim _{r \rightarrow 0} E N_{r}\left(\Gamma_{1}\right)=\lim _{r \rightarrow 0} \sum_{n} E N_{r, n}\left(\Gamma_{1}\right)
$$

As $r \rightarrow 0$ there are asymptotically $1 /\left|\Gamma_{r}\right|$ values of $n$ such that $n r+\Gamma_{r} \subset \Gamma_{1}$. Thus by stationarity we conclude that (2.7) holds and hence (2.6) holds.

Corollary 2.1. Let $P$ be as in Theorem 2.1. Then

and, if $\alpha(P)>0$,

$$
\lambda(P)=\lim _{r \rightarrow 0} \frac{1}{\left|\Delta_{r}\right|} P\left(i^{*}\left(\Delta_{r}\right)=1\right)
$$

$$
\alpha(P) / \lambda(P)=\lim _{r \rightarrow 0} E\left[N\left(\Delta_{r}\right) \mid N^{*}\left(\Lambda_{r}\right)=1\right]
$$

Proof. We obtain (2.8) by applying Theorem 2.2 directly to the random distribution $N^{*}$. Clearly (2.9) follows immediately from (2.6) and (2.8).

3. Tagged particle process. Throughout this section we consider a stationary marked process having distribution $P$ such that $0<a(P)<\infty$. Set $\Omega_{0}=$ $\{\omega: N(0)>0\}$.

Theorem 3.1. There is a unique distribution $Q$ on $\Omega$, called the tagged particle distribution, such that

$$
a(P)|\Lambda| \int Q(d \omega) f(\omega)=\int P(d \omega) \sum_{i} f\left(\omega-x_{i}\right) 1_{A}\left(x_{i}\right)
$$

for all nonnegative measurable functions $f$ on $\Omega$ and all Borel subsets $A$ of $X$ baving finite measure. $Q$ is sucb that $Q\left(\Omega_{0}\right)=1$. 
Proof. Let $f$ be a nonnegative bounded Borel function on $\Omega$. Consider the measure $\lambda_{f}$ on $X$ defined by

$$
\lambda_{f}(A)=\int P(d \omega) \sum_{i} f\left(\omega-x_{i}\right) 1_{A}\left(x_{i}\right) .
$$

It follows from the stationarity of $P$ that $\lambda_{f}(A+t)=\lambda_{f}(A)$ for all $t \in X$. Thus there is a constant $B(f)$ such that $B(f)|A|=\lambda_{f}(A)$ whenever $|A|<\infty$. Choose $|A|$ such that $0<|A|<\infty$. Then

$$
B(f)=\frac{1}{|A|} \int P(d \omega) \sum_{i} f\left(\omega-x_{i}\right) 1_{A}\left(x_{i}\right)
$$

holds for all nonnegative bounded Borel functions $f$. Thus there is a unique probability measure $Q$ on $\Omega$ such that

$$
\int Q(d \omega) f(\omega)=\frac{1}{\alpha(P)} B(f)
$$

for all such $\%$. It follows from (3.2) and (3.3) that $Q$ is the unique distribution satisfying (3.1). By setting $f=1_{\boldsymbol{\Omega}_{0}}$ in (3.1) we conclude that $Q\left(\Omega_{0}\right)=1$.

Theorem 3.2. (i) $Q$ is the unique probability measure on $\Omega$ such that for any bounded measurable function $f$ on $\Omega$ and any bounded measurable function $g$ on $X$ baving compact support

$$
\alpha(P) \int Q(d \omega) \int f(\omega+t) g(t) d t=\int P(d \omega) f(\omega) \int g(t) N(d t) .
$$

(ii) For any bounded continuous function $f$ on $\Omega$,

$$
\alpha(P) \int Q(d \omega) f(\omega)=\lim _{r \rightarrow 0} \frac{1}{\left|\Delta_{r}\right|} \int P(d \omega) f(\omega) N\left(\Delta_{r}\right) .
$$

Proof. Choose a Borel subset $A$ of $X$ such that $|A|=1$. Then

$$
\begin{aligned}
\alpha(P) \int Q(d \omega) & \int f(\omega+t) g(t) d t=\int P(d \omega) \sum_{i} \int d t f\left(\omega+t-x_{i}\right) 1_{A}\left(x_{i}\right) g(t) \\
& =\int P(d \omega) \int d s \sum_{i} f(\omega+s) 1_{A}\left(x_{i}\right) g\left(x_{i}+s\right) \\
& =\int P(d \omega) \int d s \sum_{i} f(\omega) 1_{A}\left(x_{i}-s\right) g\left(x_{i}\right) \\
& =\int P(d \omega) f(\omega) \sum_{i} g\left(x_{i}\right)=\int P(d \omega) f(\omega) \int g(t) N(d t) .
\end{aligned}
$$

This establishes (3.4), from which (3.5) follows immediately. Since (3.5) follows 
from (3.4) and uniquely determines $Q$, we conclude that (3.4) uniquely determines Q.

Corollary 3.1. Suppose $a(P)>0$ and let $f$ be a bounded continuous function on $\Omega$. Then

$$
\frac{a(P)}{\lambda(P)} \int Q(d \omega) f(\omega)=\lim _{r \rightarrow 0} E\left[f(\omega) N\left(\Delta_{r}\right) \mid N^{*}\left(\Delta_{r}\right)=1\right]
$$

and, if the particles occupy distinct sites with probability one,

$$
\int Q(d \omega) f(\omega)=\lim _{r \rightarrow 0} E\left[f(\omega) \mid N\left(\Delta_{r}\right)=1\right]
$$

This result follows immediately from Theorems 2.2 and 3.2. According to (3.7), if the particles occupy distinct sites with probability one, then $Q(A)$ can be interpreted intuitively as the "conditional probability of $A$ given that there is a particle at the origin." If $X=Z^{d}$, then this interpretation is rigorous, as can easily be seen by first letting $B=\{0\}$ in (3.5) to conclude that

$$
\alpha(P) \int Q(d \omega) f(\omega)=\int P(d \omega) f(\omega) N(0) .
$$

By letting $M$ be a set consisting of a single point we can apply the results of this section to ordinary counting measures (no marks). We will do so in the two examples below, where we compute $Q$ explicitly in some important special cases.

Example 1. Consider a random counting measure having distribution $P$ on $Z^{d}$ such that under $P\left\{\omega(x), x \in Z^{d}\right\}$ are independent and identically distributed random variables having density $b$ and mean $a, 0<a<\infty$. Then under $Q\left\{\omega(x), x \in Z^{d}\right\}$ are independent random variables such that $\omega(x)$ has density $b$ for $x \neq 0$ and $Q(\omega(0)=y)=y b(y) / a$.

This result follows immediately from equation (3.8) by considering functions $f$ of the form $f(\omega)=\Pi_{x \in J} f_{x}(\omega(x))$, where $J$ is a finite subset of $Z^{d}$.

Example 2 (compound Poisson process). Consider a Poisson process $\left\{t_{i}\right\}$ with parameter $\lambda$ on $X$. At the points $t_{i}$ we lay down $\xi_{i}$ particles, where $\left\{\xi_{i}\right\}$ are independent and identically distributed nonnegative integer-valued random variables having density $h$, mean $\mu, 0<\mu<\infty$, and $\left\{\xi_{i}\right\}$ is independent of $\left\{t_{i}\right\}$. The random counting measure $N$ on $X$ defined by $N(B)=\Sigma_{i} \xi_{i} 1_{B}\left(t_{i}\right)$, called a compound Poisson process, is a stationary random counting measure whose distribution $P$ is such that $\alpha(P)=\lambda \mu$. The corresponding tagged particle process is obtained by starting with the original compound Poisson process and independently adding $N(0)$ particles to the origin, where $Q(N(0)=y)=y b(y) / \mu$.

To verify these conclusions, we choose $A$ in (3.1) such that $|A|=1$ and set $f(\omega)=1_{\{N(0)=y\}}$. Then by $(3.1)$ 


$$
\begin{aligned}
\lambda \mu Q(N(0)=y) & =\int P(d \omega) \sum_{i} 1_{A}\left(x_{i}\right) 1_{\left\{N\left(x_{i}\right)=y\right\}}=\int P(d \omega) \sum_{i} \xi_{i} 1_{A}\left(t_{i}\right) 1_{\left\{\xi_{i}=y\right\}} \\
& =y \int P(d \omega) \sum_{i} 1_{A}\left(t_{i}\right) 1_{\left\{\xi_{i}=y\right\}}=\lambda y b(y),
\end{aligned}
$$

so that $Q(N(0)=y)=y b(y) / \mu$.

Let $A_{0}, A_{1}, \ldots, A_{n}$ be disjoint compact subsets of $X$ such that $0 \in$ int $A_{0}$. Let $f_{i}(\omega), 0 \leq i \leq n$, be bounded continuous functions of $\omega$ such that $f_{i}(\omega)$ depends only on the restriction of $\omega$ to $A_{i}$. Then for $r$ sufficiently small $\Lambda_{r} \subset A_{0}$ and hence

$$
\int P(d \omega) \prod_{i=0}^{n} f_{i}(\omega) N\left(\Delta_{r}\right)=E f_{0}(\omega) N\left(\Delta_{r}\right) \prod_{i=1}^{n} E f_{i}(\omega) .
$$

We conclude from (3.5) that

$$
Q(d \omega) \prod_{i=0}^{n} f_{i}(\omega)=\prod_{i=1}^{n} E f_{i}(\omega) \lim _{r \rightarrow 0} \frac{1}{\left|\Delta_{r}\right|} E f_{0}(\omega) N\left(\Delta_{r}\right) .
$$

This shows that under $Q$ the random variables $f_{0}(\omega), \ldots, f_{n}(\omega)$ are independently distributed and $f_{1}(\omega), \ldots, f_{n}(\omega)$ are distributed under $Q$ exactly as they are under $P$. The remainder of the results of Example 2 follow from these observations.

4. Applications of the ergodic theorem. Let $P$ be the distribution of a stationary marked point process such that $0<\alpha(P)<\infty$ and let $Q$ denote the corresponding tagged particle distribution. In this section we will obtain some applications of the multidimensional ergodic theorem.

Let $\mathcal{f}$ denote the $\sigma$-field of all Borel sets $A$ in $\Omega$ such that $1_{A}(\omega+t)=$ $1_{A}(\omega)$ with probability 1 (w.r.t. $P$ ) for every $t \in X$. Set

$$
A_{T}=\left\{x \in X:\left|x^{i}\right|<T, 1 \leq i \leq d\right\}, \quad T>0,
$$

and set $C=A_{1 / 2}$. Then $|C|=1$.

Let $Z(\omega), \omega \in \Omega$, be a random variable such that $\int P(d \omega)|Z(\omega)|<\infty$. The multidimensional pointwise ergodic theorem (see Theorem 17 of VIII.7 of Dunford and Schwartz [1]) implies that there is an $\mathcal{1}$-measurable function $\bar{Z}(\omega), \omega \in \Omega$, such that

$$
\lim _{T \rightarrow \infty} \frac{1}{\left|A_{T}\right|} \int_{A_{T}} Z(\omega-t) d t=\bar{Z}(\omega)
$$

with probability one w.r.t. $P$. It is easily seen by separating $Z$ into its positive and negative parts and using a truncation argument that (4.1) is also valid in the sense of $\mathscr{L}_{1}$ convergence w.r.t. P. Consequently $\left.\bar{Z}=E[Z \mid\}\right]$.

Set 


$$
\bar{N}=E[N(C) \mid g]=\lim _{T \rightarrow \infty} \frac{1}{\left|A_{T}\right|} \int_{A_{T}} N(C+t) d t
$$

Since

$$
\int_{A_{T-1}} N(C+t) d t \leq N\left(A_{T}\right) \leq \int_{A_{T+1}} N(C+t) d t,
$$

we conclude that

$$
\lim _{T \rightarrow \infty} \frac{N\left(A_{T}\right)}{\left|A_{T}\right|}=\bar{N}
$$

both with probability one and in $\mathscr{L}_{1}$ w.r.t. $P$. Observe that $E \bar{N}=\alpha(P)$.

Next we will show that

$$
P(\bar{N}=0)=P(N=0) .
$$

To verify (4.3), let $B$ be a relatively compact subset of $X$. By (4.1)

$$
E \lim _{T \rightarrow \infty} \frac{1}{\left|A_{T}\right|} \int_{A_{T}} 1_{\{N(B+t)=0\}}(\omega) d t=P(N(B)=0) .
$$

It is easily seen that the random variable in the left side of (4.4) equals 1 whenever $\bar{N}=0$. Thus $P(\bar{N}=0) \leq P(N(B)=0)$. Since $B$ is an arbitrary relatively compact subset of $X$, we conclude that $P(\bar{N}=0) \leq P(N(X)=0)=P(N=0)$. But clearly $P(N=0) \leq P(\bar{N}=0)$, so (4.3) holds.

Let $f$ be a bounded measurable function on $\Omega$. We conclude from (3.4) and (4.2) that

$$
\alpha(P) \lim _{T \rightarrow \infty} \frac{1}{\left|A_{T}\right|} \int Q(d \omega) \int_{A_{T}} f(\omega+t) d t=\int P(d \omega) \bar{N} f(\omega) .
$$

By letting $f$ be the indicator function of the event that the limit in (4.2) exists and is finite, we conclude from (4.5) that $\bar{N}$ exists and is finite with probability one w.r.t. $Q$. Using a similar argument we conclude that if $f$ is a bounded measurable function on $\Omega$ then

$$
\bar{f}(\omega)=\lim _{T \rightarrow \infty} \frac{1}{\left|A_{T}\right|} \int_{A_{T}} f(\omega+t) d t
$$

exists and is finite with probability one w.r.t. Q. We conclude from (4.5) that

$$
\alpha(P) \int Q(d \omega) \bar{f}(\omega)=\int P(d \omega) \bar{N} f(\omega)
$$

By letting $f$ denote the indicator function of the event $\{\bar{N}=0\}$, we conclude from (4.6) that $\bar{N} \neq 0$ with probability one w.r.t. $Q$. It also follows from (4.6) that 


$$
\alpha(P) \int Q(d \omega) \frac{\bar{f}(\omega)}{\bar{N}}=\int_{\{\bar{N} \neq 0\}} P(d \omega) f(\omega) .
$$

In particular

$$
\beta(Q)=\int Q(d \omega) \frac{1}{\bar{N}}=\frac{1}{\alpha(P)} P(N \neq 0)
$$

Let $P^{\prime}$ be the distribution of a stationary marked process such that $0<$ $a\left(P^{\prime}\right)<\infty$ and let $Q^{\prime}$ be the corresponding tagged particle distribution. It follows from (4.7) that $Q^{\prime}=Q$ if and only if $P^{\prime}$ is of the form

$$
\int P^{\prime}(d \omega) f(\omega)=c \int P(d \omega) f(\omega)+(1-c) f(0),
$$

where $0<c \leq 1 / P(N \neq 0)$. It also follows from (4.7) and (4.8) that if $Q^{\prime}=Q$, then either of the conditions $a\left(P^{\prime}\right)=a(P)$ or $P^{\prime}(N=0)=P(N=0)$ guarantees that $P^{\prime}=P$.

5. Weak convergence of processes. In the next theorem we indicate the extent to which $P$ and $Q$ depend continuously on each other.

Theorem 5.1. Let $P_{n}, n \geq 1$, be distributions of stationary marked processes sucb that $0<\alpha\left(P_{n}\right)<\infty$ and $a\left(P_{n}\right) \rightarrow c$ as $n \rightarrow \infty$, where $0<c<\infty$. Let $Q_{n}$ be the corresponding tagged particle distributions. Then there is a distribution $P$ of a stationary marked process such that $\alpha(P)=c$ and $P_{n} \rightarrow P$ weakly if and only if there is a probability distribution $Q$ on $\Omega$ such that $Q_{n} \rightarrow Q$ weakly, in which case $Q$ is the tagged particle distribution corresponding to $P$.

Proof. Suppose that $P_{n} \rightarrow P$ weakly and $\alpha\left(P_{n}\right) \rightarrow \alpha(P)$. Let $f$ be a bounded continuous function on $\Omega$ taking values in $[0,1]$ and let $g$ be a nonnegative continuous function on $X$ having compact support and such that $\int g(x) d x=1$. It follows from Theorem 3.1 that

$$
a\left(P_{n}\right) \int Q_{n}(d \omega) f(\omega)=\int P_{n}(d \omega) \sum_{i} f\left(\omega-x_{i}\right) g\left(x_{i}\right)
$$

and

$$
\alpha(P) \int Q(d \omega) f(\omega)=\int P(d \omega) \sum_{i} f\left(\omega-x_{i}\right) g\left(x_{i}\right)
$$

Since $\omega+t$ is jointly continuous in $\omega$ and $t$, we conclude that

$$
F(\omega)=\sum_{i} f\left(\omega-x_{i}\right) g\left(x_{i}\right)
$$

is a nonnegative continuous function on $\Omega$. Thus by (5.1) and (5.2) 


$$
\liminf _{n \rightarrow \infty} \int Q_{n}(d \omega) f(\omega) \geq \int Q(d \omega) f(\omega) .
$$

Replacing $f$ by $1-f$ in (5.3), we conclude that

$$
1-\lim \sup _{n \rightarrow \infty} \int Q_{n}(d \omega) f(\omega) \geq 1-\int Q(d \omega) f(\omega) .
$$

Consequently $\lim _{n \rightarrow \infty} \int Q_{n}(d \omega) f(\omega)=\int Q(d \omega) f(\omega)$, so that $Q_{n} \rightarrow Q$ weakly as $n \rightarrow \infty$.

Suppose conversely that $Q_{n} \rightarrow Q$ weakly. Let $A$ be a compact subset of $X$ and let $b$ be a bounded continuous function on $\Omega$ such that $b(\omega)=0$ whenever $N(A)=0$. Let $g$ be a nonnegative continuous function on $X$ having compact support and such that $g=1$ on $A$. Set $G(\omega)=\int g(t) N(d t)$. Set $H(\omega)=\max (G(\omega), 1)$ and $f(\omega)=b(\omega) / H(\omega)$. Then $f$ is a bounded continuous function on $\Omega$. Also

$$
f(\omega) G(\omega)=b(\omega), \quad \omega \in \Omega .
$$

Equation (5.5) is obvious if $G(\omega) \geq 1$. If $G(\omega)<1$, then $1>G(\omega) \geq \int_{A} g(t) N(d t) \geq$ $N(A)$. Thus $N(A)=0$ and hence $f(\omega)=b(\omega)=0$. Thus (5.5) holds also if $G(\omega)<1$.

By (5.5) and Theorem 3.2

$$
\alpha\left(P_{n}\right) \int Q_{n}(d \omega) \int \frac{b(\omega+t) g(t)}{H(\omega+t)} d t=\int P_{n}(d \omega) b(\omega) .
$$

Thus

$$
\lim _{n \rightarrow \infty} \int P_{n}(d \omega) b(\omega)=c \int Q(d \omega) \int \frac{b(\omega+t) g(t)}{H(\omega+t)} d t
$$

It follows from (5.6) and Theorem 2.3 of Harris [3] that there is a probability measure $P$ on $\Omega$ such that

$$
\lim _{n \rightarrow \infty} \int P_{n}(d \omega) b(\omega)=\int P(d \omega) b(\omega)
$$

for all such functions $b$. From (5.7) and Theorem 2.1 of Harris [3] we now conclude that $P_{n} \rightarrow P$ weakly. Since $P_{n}$ is stationary, so is $P$.

It remains only to show that $a(P)=c$, since the first half of the proof the theorem then implies that $Q$ is the tagged particle distribution corresponding to $P$.

From (5.6) and (5.7)

$$
\int P(d \omega) b(\omega)=c \int Q(d \omega) \int \frac{b(\omega+t) g(t)}{H(\omega+t)} d t .
$$

We can apply this formula when $b(\omega)=N(A)$ for some compact subset $A$ of $X$ having positive measure and $g=1_{A}$. Then $b(\omega+t)=N(A-t)$ and $H(\omega+t)=$ $\max (N(A-t), 1)$. Thus $(5.8)$ becomes 


$$
\int P(d \omega) N(A)=c \int Q(d \omega) \int_{A} \frac{N(A-t)}{\max (N(A-t), 1)} d t
$$

Since each $Q_{n}$ assigns probability one to the set $\{N(0) \geq 1\}$, so does $Q$. Thus with $Q$-probability $1, N(A-t) \geq 1, t \in A$, and hence (5.9) reduces to $\int P(d \omega) N(A)=$ $c|A|$, which implies that $a(P)=c$ as desired.

6. Marked motion process. A marked motion counting measure $\psi$ is an integervalued measure on $X \times M \times X \times M$ such that $\psi(A \times M \times X \times M)<\infty$ and $\psi(X \times M \times A \times M)<\infty$ for all compact subsets $A$ of $X$. Associated with $\psi$ is its realization as a sequence of points $\left\{\left(x_{i}, m_{i}, x_{i}^{\prime}, m_{i}^{\prime}\right)\right\}$ such that

$$
\iiint \int \psi\left(d x, d m, d x^{\prime}, d m^{\prime}\right) f\left(x, m, x^{\prime}, m^{\prime}\right)=\sum_{i} f\left(x_{i}, m_{i}, x_{i}^{\prime}, m_{i}^{\prime}\right) .
$$

Let $\Psi$ be the collection of all such measures $\psi$. We can make $\Psi$ into a complete separable metric space in such a manner that $\psi_{n} \rightarrow \psi$ if and only if $\lim _{n \rightarrow \infty} \int f d \psi_{n}=\int f d \psi$ for all bounded continuous functions $f\left(x, m, x^{\prime}, m\right)$ which either vanish for $x$ outside of some compact set or vanish for $x^{\prime}$ outside of some compact set. By a measurable subset of $\Psi$ we mean a Borel set.

For $\psi \in \Psi$ and $t \in X$ define $\psi+t \in \Psi$ by

$$
\begin{aligned}
\iiint \int(\psi+t) & \left(d x, d m, d x^{\prime}, d m^{\prime}\right) f\left(x, m, x^{\prime}, m^{\prime}\right) \\
= & \iiint \int \psi\left(d x, d m, d x^{\prime}, d m^{\prime}\right) f\left(x+t, m, x^{\prime}+t, m^{\prime}\right) .
\end{aligned}
$$

Then $\psi+t$ is jointly continuous in $\psi$ and $t$. The measure $\psi$ gives rise to marginal marked counting measures $\omega$ and $\omega^{\prime}$ on $X \times M$ defined by

$$
\omega(B)=\psi(B \times X \times M) \text { and } \omega^{\prime}(B)=\psi(X \times M \times B) .
$$

Both $\omega$ and $\omega^{\prime}$ depend continuously on $\psi$.

By a marked motion process we mean a random marked motion counting measure $\psi$ distributed according to a probability measure $R$ on $\Psi$. We think of the corresponding random marked counting measures $\omega$ and $\omega^{\prime}$ as the marked processes at times 0 and 1 respectively. The distribution $R$ gives rise to the marginal distributions $P$ and $P^{\prime}$ of $\omega$ and $\omega^{\prime}$ respectively. The marked motion process and its distribution $R$ are called translation invariant if $\int R(d \psi) f(\psi+t)=\int R(d \psi) f(\psi)$ for all bounded measurable functions $f$ on $\Psi$ and all $t \in X$. If $R$ is translation invariant, then $P$ and $P^{\prime}$ are stationary.

Theorem 6.1. If $P$ and $P^{\prime}$ are the marginal distributions corresponding to a translation invariant marked motion process, then $\alpha(P)=\alpha\left(P^{\prime}\right)$.

Proof. Set $A=\left\{x \in X: 0 \leq x^{i}<1,1 \leq i \leq d\right\}$. Then $A+n, n \in Z^{d}$, is a 
partition of $X$ into disjoint sets. By translation invariance for $n \in Z^{d}$,

$$
\int R(d \psi) \psi(A \times M \times(A+n) \times M)=\int R(d \psi) \psi((A-n) \times M \times A \times M) .
$$

By summing on $n \in Z^{d}$ we conclude that

$$
\int R(d \psi) \psi(A \times M \times X \times M)=\int R(d \psi) \psi(X \times M \times A \times M)
$$

or equivalently that

$$
\int P(d \omega) \omega(A \times M)=\int P^{\prime}\left(d \omega^{\prime}\right) \omega^{\prime}(A \times M) .
$$

This shows that $\alpha(P)=\alpha\left(P^{\prime}\right)$.

We assume throughout the remainder of this section that $R$ is translation invariant and that $0<a(P)=\alpha\left(P^{\prime}\right)<\infty$. Let $Q$ and $Q^{\prime}$ be the tagged particle distributions corresponding to $P$ and $P^{\prime}$. Then $Q^{\prime}$ is the distribution of $\omega^{\prime}$ viewed from a particle selected "at random" at time 1 . We will now determine several ways in which $Q^{\prime}$ can be considered the distribution of $\omega^{\prime}$ viewed from the position at time 1 of a particle selected "at random" at time 0.

Theorem 6.2. There is a unique distribution $\widetilde{Q}$ on $\Omega$ such that

$$
a(P)|A| \int \widetilde{Q}\left(d \omega^{\prime}\right) f\left(\omega^{\prime}\right)=\int R(d \psi) \sum_{i} f\left(\omega^{\prime}-x_{i}^{\prime}\right) 1_{A}\left(x_{i}\right)
$$

for all bounded measurable functions $f$ on $\Omega$ and all Borel subsets $A$ of $X$ baving finite measure.

The proof of this theorem is the same as that of Theorem 3.1.

Theorem 6.3. $\widetilde{Q}=Q^{\prime}$.

Proof. Let $A$ be a subset of $X$ such that $|A|=1$. Let $B$ be a Borel subset of $X$ and $f$ a bounded measurable function on $\Omega$. Then

$$
\begin{aligned}
\alpha(P) \int \tilde{Q}\left(d \omega^{\prime}\right) & \int_{B} f\left(\omega^{\prime}+t\right) d t=\int R(d \psi) \sum_{i} \int f\left(\omega^{\prime}+t-x_{i}^{\prime}\right) 1_{A}\left(x_{i}\right) 1_{B}(t) d t \\
& =\int R(d \psi) \sum_{i} \int f\left(\omega^{\prime}+s\right) 1_{A}\left(x_{i}\right) 1_{B}\left(s+x_{i}^{\prime}\right) d s \\
& =\int R(d \psi) f\left(\omega^{\prime}\right) \sum_{i} \int 1_{A}\left(x_{i}-s\right) 1_{B}\left(x_{i}^{\prime}\right) d s \\
& =\int R(d \psi) f\left(\omega^{\prime}\right) N^{\prime}(B)=\int P^{\prime}\left(d \omega^{\prime}\right) f\left(\omega^{\prime}\right) N^{\prime}(B) .
\end{aligned}
$$

Thus by Theorem 3.2 we conclude that $\tilde{Q}=Q^{\prime}$.

Theorem 6.4. There is a family $S(\omega, d \psi), \omega \in \Omega$, of probability measures on $\Psi$ baving the following properties: 
(i) $S\left(\omega_{0},\left\{\psi \mid \omega(\psi)=\omega_{0}\right\}\right)=1, \omega_{0} \in \Omega$.

(ii) $S(\omega, B)$ is measurable in $\omega$ for eacb measurable subset $B$ of $\Psi$.

(iii) $S(\omega+t, B+t)=S(\omega, B)$.

(iv) If $f$ is a nonnegative measurable function on $\Psi$, then

$$
\int R(d \psi) f(\psi)=\int P(d \omega) \int S(\omega, d \psi) f(\psi) .
$$

Proof. See $\$ 8$ of Harris [3]. (With respect to (iii), Harris showed only that, for fixed $t$ and $B$, it holds with probability one. However, by using the fact that $\Psi$ is a complete separable metric space, it is straightforward but a little tedious to show that there is a version so that (iii) holds as stated. See Appendix 2 of [3] for similar arguments.)

By definition, $S(\omega,$.$) is the conditional distribution of \psi$ given $\omega$, when $\psi$ is distributed according to $R$. More generally, we can use $S(\omega,$.$) together with$ any initial distribution of $\omega$ to define a distribution on $\Psi$. In the next theorem we will do this, letting $\omega$ be chosen initially according to $Q$. We will show that the distribution of $\omega^{\prime}$ viewed from the position at time 1 of a particle chosen at random from those at the origin at time 0 is just $Q^{\prime}$.

Theorem 6.5. For any bounded measurable function $f$ on $\Omega$,

$$
\int Q(d \omega) \int s(\omega, d \psi) \frac{1}{N(0)} \sum_{i} f\left(\omega^{\prime}-x_{i}^{\prime}\right) 1_{\{0\}}\left(x_{i}\right)=\int Q^{\prime}\left(d \omega^{\prime}\right) f\left(\omega^{\prime}\right) .
$$

Proof. Set $g(\omega)=\int S(\omega, d \psi)(1 / N(0)) \Sigma_{i} f\left(\omega^{\prime}-x_{i}^{\prime}\right) 1_{\{0\}}\left(x_{i}\right)$. Then

$$
\begin{aligned}
g(\omega-c) & =\int s(\omega-c, d \psi) \frac{1}{N(c)} \sum_{i} f\left(\omega^{\prime}-x_{i}^{\prime}\right) 1_{\{0\}}\left(x_{i}-c\right) \\
& =\int s(\omega, d \psi) \frac{1}{N(c)} \sum_{i} f\left(\omega^{\prime}-x_{i}^{\prime}\right) 1_{\{0\}}\left(x_{i}-c\right) .
\end{aligned}
$$

Let $A$ be a subset of $X$ such that $|A|=1$. By Theorem 3.1 the left side of (6.2) equals

$$
\begin{aligned}
\frac{1}{\alpha(P)} \int P(d \omega) & \sum_{j} g\left(\omega-x_{j}\right) 1_{A}\left(x_{j}\right) \\
& =\frac{1}{\alpha(P)} \int P(d \omega) \sum_{j} 1_{A}\left(x_{j}\right) \int S(\omega, d \psi) \frac{1}{N\left(x_{j}\right)} \sum_{i} f\left(\omega^{\prime}-x_{i}^{\prime}\right) 1_{\{0\}}\left(x_{i}-x_{j}\right) \\
& =\frac{1}{\alpha(P)} \int P(d \omega) \int S(\omega, d \psi) \sum_{i, j} \frac{1}{N\left(x_{j}\right)} 1_{A}\left(x_{j}\right) f\left(\omega^{\prime}-x_{i}^{\prime}\right) 1_{\{0\}}\left(x_{i}-x_{j}\right) \\
& =\frac{1}{\alpha(P)} \int R(d \psi) \sum_{i, j} \frac{1}{N\left(x_{i}\right)} 1_{A}\left(x_{i}\right) f\left(\omega^{\prime}-x_{i}^{\prime}\right) 1_{\{0\}}\left(x_{i}-x_{j}\right) \\
& =\frac{1}{\alpha(P)} \int R(d \psi) \sum_{i} 1_{A}\left(x_{i}\right) f\left(\omega^{\prime}-x_{i}^{\prime}\right)=\int \widetilde{Q}\left(d \omega^{\prime}\right) f\left(\omega^{\prime}\right)=\int Q^{\prime}\left(d \omega^{\prime}\right) f\left(\omega^{\prime}\right)
\end{aligned}
$$


as desired.

We conclude this section by describing some continuity properties of motions corresponding to independent translations. These results will be used in $\$ 8$.

Theorem 6.6. Let $S(\omega, d \psi)$ be such that if $\omega=\left\{\left(x_{i}, m_{i}\right)\right\}$, then $\psi$ is distributed as $\left\{\left(x_{i}, m_{i}, x_{i}+w_{i}, m_{i}\right)\right\}$, where $\left\{w_{i}\right\}$ are independent and identically dis. tributed random variables which are independent of $\left\{\left(x_{i}, m_{i}\right)\right\}$. Then $S(\omega, d \psi)$ depends continuously on $\omega$ (in the sense of weak convergence of measures).

Proof. Let $\omega_{n} \rightarrow \omega$. We suppose that $\omega_{n}(X \times M)=\omega(X \times M)=\infty$, the modifications necessary to handle the other cases being straightforward. We can suppose that

$$
\omega_{n}=\left\{\left(x_{i}^{(n)}, m_{i}^{(n)}\right)\right\} \text { and } \omega=\left\{\left(x_{i}, m_{i}\right)\right\}
$$

where $x_{i}^{(n)} \rightarrow x_{i}$ and $m_{i}^{(n)} \rightarrow m_{i}$ as $n \rightarrow \infty$. The distributions $S\left(\omega_{n}, d \psi\right)$ and $S(\omega, d \psi)$ correspond respectively to the distributions of

$$
\psi_{n}=\left\{\left(x_{i}^{(n)}, m_{i}^{(n)}, x_{i}^{(n)}+w_{i}, m_{i}^{(n)}\right)\right\} \quad \text { and } \quad \psi=\left\{\left(x_{i}, m_{i}, x_{i}+w_{i}, m_{i}\right)\right\} .
$$

But $\psi_{n} \rightarrow \psi$ with probability one as $n \rightarrow \infty$ and hence the distribution.of $\psi_{n}$ converges weakly to that of $\psi$.

Corollary 6.1. Let $S(\omega, d \psi)$ be as in Theorem 6.6. For anydistribution $P$ on $\Omega$ define the distribution $P^{\prime}$ on $\Omega$ by the formula

$$
\int P^{\prime}\left(d \omega^{\prime}\right) f\left(\omega^{\prime}\right)=\int P(d \omega) \int s(\omega, d \psi) f\left(\omega^{\prime}\right) .
$$

Then $P^{\prime}$ depends continuously on $P$ (in the sense of weak convergence of measures).

7. One-dimensional case. We will now assume that $d=1$ and hence $X=Z$ or $X=R$. We will consider a stationary marked process having distribution $P$ such that $0<\alpha(P)<\infty$ and $P(N(X)>0)=1$. By Theorem 2.1 we can consider $P$ defined on the Borel sets of

$$
\Omega^{*}=\{\omega: N((\infty, 0))=N((0, \infty))=\infty\} .
$$

Closely related to the tagged particle distribution $Q$ is a "spacing process" having distribution $Q_{0}$. In this section, we will obtain closed form expressions relating $P, Q$, and $Q_{0}$ to each other and show that these distributions depend continuously on each other.

Let $\Xi$ denote the collection of all doubly infinite sequences $\xi$ of elements $\xi_{i}=\left(\eta_{i}, m_{i}\right)$ in $X^{+} \times M$, where $X^{+}=\{x \in X: x \geq 0\}$, such that $\Sigma_{-\infty}^{0} \eta_{i}=$ $\Sigma_{0}^{\infty} \eta_{i}=\infty$. For the measurable sets on $\Xi$ take the product $\sigma$-field. For $\xi \in \Xi$ we let $\eta=\eta(\xi)$ denote the doubly infinite sequence of elements $\eta_{i}$. The shift 
$S: \Xi \rightarrow \Xi$ is defined by $(s \xi)_{i}=\xi_{i+1}=\left(\eta_{i+1}, m_{i+1}\right)$.

Let $\omega \in \Omega^{*}$. Then $\omega$ corresponds to the sequence $\left\{\left(x_{i}, m_{i}\right)\right\}_{-\infty}^{\infty}$ of elements of $X \times M$. We can assume that $x_{i+1} \geq x_{i}$ for all $i, x_{0} \leq 0$, and $x_{1}>0$. Clearly, $\lim _{i \rightarrow-\infty} x_{i}=-\infty$ and $\lim _{i \rightarrow \infty} x_{i}=\infty$ since there are only a finite number of the $x_{i}$ 's in any compact subset of $X$. Set $\eta_{i}(\omega)=x_{i+1}-x_{i}, \xi_{i}(\omega)=\left(\eta_{i}(\omega), m_{i}(\omega)\right)$, and $\xi(\omega)=\left\{\xi_{i}(\omega)\right\}_{-\infty}^{\infty}$. Observe that $\eta_{0}(\omega)>0, \omega \in \Omega^{*}$.

For $\xi=\left\{\left(\eta_{i}, m_{i}\right)\right\}_{-\infty}^{\infty}$ in $\Xi$, let $\omega(\xi)$ be the element in $\Omega^{*}$ corresponding to $\left\{\left(\eta_{0}+\cdots+\eta_{i-1}, m_{i}\right)\right\}_{-\infty}^{\infty}$. Here $\eta_{0}+\cdots+\eta_{i-1}=-\left(\eta_{i}+\cdots+\eta_{-1}\right), i \leq-1$, and $\eta_{0}+\cdots+\eta_{-1}=0$. Observe that $\omega(\xi) \in \Omega_{0}^{*}$, where $\Omega_{0}^{*}=\left\{\omega \in \Omega^{*}: N(0)>0\right\}$.

Observe also that

and

$$
\begin{gathered}
\omega(\xi(\omega))=\omega, \quad \omega \in \Omega_{0}^{*}, \\
\xi(\omega(\xi))=\xi \text { if and only if } \eta_{0}(\xi)>0,
\end{gathered}
$$

Theorem 7.1. Let $\omega_{0} \in \Omega_{0}^{*}$ and set $N(0)=N\left(0, \omega_{0}\right)$. Then

$$
\left\{\xi \in \Xi: \omega(\xi)=\omega_{0}\right\}=\left\{S^{-i} \xi\left(\omega_{0}\right): 0 \leq i \leq N(0)-1\right\} .
$$

Proof. Observe that $x_{-N(0)}\left(\omega_{0}\right)<0$ and $x_{-i}\left(\omega_{0}\right)=0,0 \leq i \leq N(0)-1$. Consequently, $\eta_{N(0)}>0$ and $\eta_{-i}\left(\omega_{0}\right)=0,0<i \leq N(0)-1$. By $(7.1) \omega\left(\xi\left(\omega_{0}\right)\right)=\omega_{0}$. Choose $i$ such that $0<i \leq N(0)-1$. Then

$$
\eta_{0}\left(\omega_{0}\right)+\cdots+\eta_{-i-1}\left(\omega_{0}\right)=-\left[\eta_{-i}\left(\omega_{0}\right)+\cdots+\eta_{-1}\left(\omega_{0}\right)\right]=0
$$

and hence by (7.1) and (7.3) $\omega\left(S^{-i} \xi\left(\omega_{0}\right)\right)=\omega\left(\xi\left(\omega_{0}\right)\right)=\omega_{0}$.

Suppose conversely that $\xi \in \Xi$ and $\omega(\xi)=\omega_{0}$. Set

$$
i=\max \left\{j: \eta_{0}+\cdots+\eta_{j-1}=0\right\} \text {. }
$$

Then $0 \leq i \leq N(0)-1$ and $\omega\left(S^{i} \xi\right)=\omega(\xi)$. Also $\eta_{0}\left(S^{i} \xi\right)>0$. Thus by (7.2) $\xi\left(\omega\left(S^{i} \xi\right)\right)=S^{i} \xi$. Consequently, $\xi\left(\omega_{0}\right)=\xi(\omega(\xi))=\xi\left(\omega\left(S^{i} \xi\right)\right)=S^{i} \xi$ and hence $\xi=$ $S^{-i} \xi\left(\omega_{0}\right)$. This completes the proof of the theorem.

A probability distribution on $\Xi$ is called stationary if $S$ is measure preserving with respect to this distribution. Stimulated by the results of Ryll-Nardzewski [4] we would like to define a distribution $Q_{0}$ on $\Xi$ which is stationary and corresponds to $Q$ in a natural way.

If $Q(N(0)>1)=0$, we can let $Q_{0}$ be the distribution of $\xi(\omega)$ when $\omega$ is distributed according to $Q$. If, $Q(N(0)>1)>0$, however, then $\xi(\omega)$ is not stationary. 
To see this we need only observe that $Q\left(\eta_{0}(\omega)=0\right)=0$ but $Q\left(\eta_{-1}(\omega)=0\right)=$ $Q(N(0)>1)>0$. A reasonable guess is to choose $\xi(\omega)$ at random from the points in $\{\xi \in \Xi: \omega(\xi)=\omega\}$. Led by this reasoning and Theorem 7.1 we define the spacing distribution $Q_{0}$ by

$$
\int Q_{0}(d \xi) g(\xi)=\int Q(d \omega) \frac{1}{N(0)} \sum_{i=0}^{N(0)-1} g\left(S^{-i} \xi(\omega)\right),
$$

for $g$ a bounded measurable function on $\Xi$.

Theorem 7.2. The shift operator $S$ is measure preserving with respect to the spacing distributions $Q_{0}$.

As a first step in proving this theorem we will obtain a result that is itself interesting.

Lemma 7.1. Let $f$ be a bounded measurable function on $\Omega_{0}^{*}$. Then

$$
\int Q(d \omega) \frac{f(\omega-x-N(0)}{N(0)}=\int Q(d \omega) \frac{f(\omega)}{N(0)} .
$$

Proof of Lemma 7.1. Consider the motion process $\left\{\left(x_{i}, m_{i}, x_{i-1}, m_{i-1}\right)\right\}$. Since $\omega^{\prime}=\omega$ with probability one we conclude that $P^{\prime}=P$ and hence $Q^{\prime}=Q$. Thus by Theorem 6.5

$$
\int Q(d \omega) \frac{1}{N(0)} \sum_{i} f\left(\omega-x_{i-1}\right) 1_{\{0\}}\left(x_{i}\right)=Q(d \omega) f(\omega) .
$$

Now $x_{-(N(0)-1)}=\cdots=x_{0}=0$ and $x_{-N(0)}<0$, so

$$
\int Q(d \omega) \frac{1}{N(0)}\left[(N(0)-1) f(\omega)+f(\omega-x-N(0)]=\int Q(d \omega) f(\omega),\right.
$$

from which (7.4) follows immediately.

Proof of Theorem 7.2. By setting $f(\omega)=g(\xi(\omega))$ in (7.4) we conclude that

$$
\int Q(d \omega) \frac{g(\xi(\omega-x-N(0))}{N(0)}=\int Q(d \omega) \frac{g(\xi(\omega))}{N(0)} .
$$

By definition

$$
\int Q_{0}(d \xi) g(\xi)=\int Q(d \omega) \frac{1}{N(0)} \sum_{i=0}^{N(0)-1} g\left(S^{-i} \xi(\omega)\right)
$$

and hence 


$$
\int Q_{0}(d \xi) g\left(S^{-1} \xi\right)=\int Q(d \omega) \frac{1}{N(0)} \sum_{i=0}^{N(0)} g\left(S^{-i} \xi(\omega)\right)
$$

Consequently,

$$
\int Q_{0}(d \xi)\left(g\left(S^{-1} \xi\right)-g(\xi)\right)=\int Q(d \omega) \frac{g\left(S^{-N(0)} \xi(\omega)\right)-g(\xi(\omega))}{N(0)} .
$$

Observe that

$$
S^{-N(0)} \xi(\omega)=\xi\left(\omega-x_{-N(0)}\right) .
$$

From (7.5)-(7.7) we conclude that the left side of (7.6) equals zero, which proves the theorem.

For $\omega \in \Omega^{*}$, let $\left\{t_{i}\right\}_{-\infty}^{\infty}$ be the atoms of the measure $N$ on $X$ arranged in increasing order so that $t_{0}=x_{0}$. Then for $A$ a Borel subset of $X, N(A)=$ $\Sigma_{i} N\left(t_{i}\right) 1_{A}\left(t_{i}\right)$. The following theorem provides a characterization of the tagged particle distribution $Q$. It says that $Q(d \omega) / N(0)$ is stationary in an appropriate sense.

Theorem 7.3. For any bounded measurable function $f$ on $\Omega_{0}^{*}$,

$$
\int Q(d \omega) \frac{f\left(\omega-t_{i}\right)}{N(0)}=\int Q(d \omega) \frac{f(\omega)}{N(0)}, \quad-\infty<i<\infty .
$$

Proof. Observe that $x_{-N(0)}=t_{-1}$. Thus by Lemma 7.1 for any bounded measurable function $b$ on $\Omega_{0}^{*}$,

$$
\int Q(d \omega) \frac{b(\omega-t-1(\omega))}{N(0)} \equiv \int Q(d \omega) \frac{b(\omega)}{N(0)} .
$$

Set $b(\omega)=f\left(\omega-t_{i}(\omega)\right)$. Since $t_{i}\left(\omega-t_{-1}(\omega)\right)=t_{i-1}(\omega)-t_{-1}(\omega)$, we conclude that $b\left(\omega-t_{-1}(\omega)\right)=f\left(\omega-t_{i-1}(\omega)\right)$. Thus (7.9) becomes

$$
\int Q(d \omega) \frac{f\left(\omega-t_{i-1}\right)}{N(0)}=\int Q(d \omega) \frac{f\left(\omega-t_{i}\right)}{N(0)},
$$

which implies that (7.8) holds.

Theorem 7.4. Let $g$ be a bounded or nonnegative measurable function on $\Xi$ and let $A$ be a subset of $X$ such that $0<|A|<\infty$. Then

$$
\alpha(P)|A| \int Q_{0}(d \xi) g(\xi)=\int P(d \omega) \sum_{i} 1_{A}\left(x_{i}\right) g\left(S^{-i} \xi(\omega)\right) .
$$


Proof. It follows from Theorem 3.1 and the definition of $Q_{0}$ that

$$
\alpha(P)|A| \int Q_{0}(d \xi) g(\xi)=\int P(d \omega) \sum_{i} 1_{A}\left(x_{i}\right) \frac{1}{N\left(x_{i}\right)} \sum_{j=0}^{N\left(x_{i}\right)-1} g\left(S^{-j} \xi\left(\omega-x_{i}\right)\right) \text {. }
$$

Let $i_{k}$ be defined by $i_{1}=1$ and $i_{k+1}-i_{k}=N\left(x_{i_{k}}\right)$. Then $x_{i_{k}+j}=x_{i_{k}}>x_{i_{k}-1}$, $0 \leq j \leq N\left(x_{i_{k}}\right)-1$, and hence

$$
S^{-j} \xi\left(\omega-x_{i_{k}}\right)=S^{-i S^{-i} k} \xi(\omega)=S^{-i_{k}-j} \xi(\omega), \quad 0 \leq j \leq N\left(x_{i_{k}}\right)-1 .
$$

We conclude that

$$
\begin{gathered}
\sum_{i} 1_{A}\left(x_{i}\right) \frac{1}{N\left(x_{i}\right)} \sum_{j=0}^{N\left(x_{i}\right)-1} g\left(S^{-i} \xi\left(\omega-x_{i}\right)\right)=\sum_{k} 1_{A}\left(x_{i_{k}}\right) \sum_{j=0}^{N\left(x_{i}\right)-1} g\left(S^{-j} \xi\left(\omega-x_{i_{k}}\right)\right) \\
=\sum_{k} 1_{A}\left(x_{i_{k}}\right) \sum_{j=0}^{i_{k+1}{ }^{-i} k^{-1}} g\left(S^{-i_{k}-j} \xi(\omega)\right)=\sum_{k} \sum_{i=i_{k}}^{i_{k+1}-1} 1_{A}\left(x_{i}\right) g\left(S^{-i} \xi(\omega)\right) \\
=\sum_{i} 1_{A}\left(x_{i}\right) g\left(S^{-i} \xi(\omega)\right),
\end{gathered}
$$

from which the theorem follows.

We will now derive a series of formulas for computing $P, Q$, and $Q_{0}$ in terms of each other.

Theorem 7.5. Let $f$ be a bounded or nonnegative measurable function on $\Omega^{*}$ and let $g$ be a bounded or nonnegative measurable function on $\Xi$. Then

$$
\begin{aligned}
\int P(d \omega) f(\omega) & =a(P) \int Q(d \omega) \frac{1}{N(0)} \int_{0}^{\eta} 0 f(\omega-t) d t \\
\alpha(P) \int Q(d \omega) f(\omega) & =\int P(d \omega) f\left(\omega-x_{0}\right) \frac{N\left(x_{0}\right)}{\eta_{0}} ; \\
\int Q_{0}(d \xi) g(\xi) & =\int Q(d \omega) \frac{1}{N(0)} \sum_{i=0}^{N(0)-1} g\left(S^{-i} \xi(\omega)\right) ; \\
\int Q(d \omega) f(\omega) & \left.=\int Q_{0}(d \xi) f\left(c^{\prime}\right)(\xi)\right) ; \\
\alpha(P) \int Q_{0}(d \xi) g(\xi) & =\int P(d \omega) \frac{1}{\eta_{0}} \sum_{i=0}^{N\left(x_{0}\right)-1} g\left(S^{-i} \xi(\omega)\right) ; \\
\int P(d \omega) f(\omega) & =\alpha(P) \int Q_{0}(d \xi) \int_{0}^{\eta}{ }^{(\xi)} f(\omega(\xi)-t) d t .
\end{aligned}
$$


Proof. Let $A$ be a subset of $X$ such that $|A|=1$. By Theorem 3.1

$$
\begin{aligned}
\alpha(P) & \int Q(d \omega) \int_{0}^{\eta} 0^{(\omega)} b(\omega-t) d t=\int P(d \omega) \sum_{i} \int_{0}^{\eta} 0^{\left(\omega-x_{i}\right)} b\left(\omega-t-x_{i}\right) 1_{A}\left(x_{i}\right) d t \\
& =\int P(d \omega) \sum_{i} \int b\left(\omega-t-x_{i}\right) 1_{\left[0, \eta_{0}\left(\omega-x_{i}\right)\right)}(t) 1_{A}\left(x_{i}\right) d t \\
& =\int P(d \omega) \int \sum_{i} b(\omega-s) 1_{\left[0, \eta_{0}\left(\omega-x_{i}\right)\right)}\left(s-x_{i}\right) 1_{A}\left(x_{i}\right) d s \\
& \left.=\int P(d \omega) \int \sum_{i} b(\omega) 1_{\left[0, \eta_{0}\right.}\left(\omega_{+} s-x_{i}(\omega+s)\right)\right) \\
& =\int P(d \omega) b(\omega) \int \sum_{i} 1_{\left[0, \eta_{0}\left(\omega-x_{i}\right)\right)}\left(-x_{i}\right) 1_{A}\left(x_{i}+s\right) d s \\
& =\int P(d \omega) b(\omega) \sum_{i} 1_{\left\{x_{i}=x_{0}\right\}}\left(x_{i}(\omega+s)\right) d s
\end{aligned}
$$

In summary

$$
\alpha(P) \int Q(d \omega) \int_{0}^{\eta} 0^{(\omega)} b(\omega-t) d t=\int P(d \omega) b(\omega) N\left(x_{0}\right)
$$

Set $b(\omega)=f(\omega) / N\left(x_{0}\right)$ in (7.16). Since $b(\omega-t)=f(\omega-t) / N\left(x_{0}\right), \omega \in \Omega_{0}^{*}$ and $0 \leq t<\eta_{0}(\omega)$, (7.10) follows.

By setting $b(\omega)=f\left(\omega-x_{0}\right) / \eta_{0}$ in (7.16), we obtain (7.11). Equation (7.12) is true by definition.

Setting $g(\xi)=f(\omega(\xi))$ in (7.12), and using Theorem 7.1, we find that

$$
\int Q_{0}(d \xi) f(\omega(\xi))=\int Q(d \omega) \frac{1}{N(0)} \sum_{i=0}^{N(0)-1} f\left(\omega\left(S^{-i} \xi(\omega)\right)\right)=\int Q(d \omega) f(\omega),
$$

which proves (7.13).

To prove (7.14) we set $f(\omega)=(1 / N(0)) \sum_{i=0}^{N(0)-1} g\left(S^{-i} \xi(\omega)\right)$ in (7.11) and use (7.12) to conclude that

$$
\begin{aligned}
& \alpha(P) \int Q_{0}(d \xi) g(\xi)=\alpha(P) \int Q(d \omega) \frac{1}{N(0)} \sum_{i=0}^{N(0)-1} g\left(S^{-i} \xi(\omega)\right) \\
& \quad=\int P(d \omega) \frac{1}{\eta_{0}} \sum_{i=0}^{N\left(x_{0}\right)-1} g\left(S^{-i} \xi\left(\omega-x_{0}\right)\right)=\int P(d \omega) \frac{1}{\eta_{0}} \sum_{i=0}^{N\left(x_{0}\right)-1} g\left(S^{-i} \xi(\omega)\right) .
\end{aligned}
$$

To prove (7.15) we set $g(\xi)=\int_{0}^{\eta_{0}(\xi)} f(\omega(\xi)-t) d t$ in (7.14) and obtain

$$
\begin{aligned}
& \alpha(P) \int Q_{0}(d \xi) \int_{0}^{\eta} 0^{(\xi)} f(\omega(\xi)-t) d t \\
& \quad=\int P(d \omega) \frac{1}{\eta_{0}} \sum_{i=0}^{N\left(x_{0}\right)-1} \int_{0}^{\eta_{0}{ }^{\left(S^{-i} \xi(\omega)\right)} f\left(\omega\left(S^{-i} \xi(\omega)\right)-t\right) d t .}
\end{aligned}
$$


Observe that $\omega(\xi))=\omega-x_{0}(\omega)$ and

$$
\begin{aligned}
\eta_{0}\left(s^{-i} \xi(\omega)\right) & =\eta_{0}(\omega), & & i=0, \\
& =0, & & 0<i<N\left(x_{0}\right)-1 .
\end{aligned}
$$

Thus by (7.17)

$$
\begin{aligned}
& a(P) \int Q_{0}(d \xi) \int_{0}^{\eta_{0}(\xi)} f(\omega(\xi)-t) d t=\int P(d \omega) \frac{1}{\eta_{0}} \int_{0}^{\eta_{0}(\omega)} f\left(\omega-x_{0}-t\right) d t \\
& =\int P(d \omega) \int \frac{1}{x_{1}-x_{0}} 1_{\left[x_{0}, x_{1}\right]}(s) f(\omega-s) d s \\
& =\int P(d \omega) f(\omega) \int \frac{{ }^{1}\left[x_{0}\left(\omega_{+}\right), x_{1}\left(\omega_{+} s\right)\right]}{(s)} \frac{x_{1}(\omega+s)-x_{0}(\omega+s)}{x^{2}} d s=\int P(d \omega) f(\omega),
\end{aligned}
$$

as desired.

Corollary 7.1. The following formulas involving $a(P)$ are valid:

$$
\begin{aligned}
& \int P(d \omega) \frac{N\left(x_{0}\right)}{\eta_{0}}=\alpha(P) \\
& \int Q(d \omega) \frac{\eta_{0}}{N(0)}=\frac{1}{\alpha(P)} ; \\
& \int Q_{0}(d \xi) \eta_{0}(\xi)=\frac{1}{\alpha(\bar{P})}
\end{aligned}
$$

Proof. Formula (7.18) follows by taking $\dot{f}=1$ in $(7.11)$; (7.19) follows by taking $f=1$ in (7.10); and (7.20) follows by taking $f=1$ in (7.15).

Let $\mathcal{P}$ denote the collection of all stationary distributions $P$ on $\Omega^{*}$ such that $\alpha(P)<\infty$. We topologize $\mathcal{P}$ such that $P_{n} \rightarrow P$ if and only if $P_{n}$ converges weakly to $P$ and $\alpha\left(P_{n}\right) \rightarrow \alpha(P)$ as $n \rightarrow \infty$.

Let 2 denote the collection of all probability measures $Q$ on $\Omega_{0}^{*}$ such that $\beta(Q)=\int Q(d \omega)\left(\eta_{0} / N(0)\right)<\infty$, and for all nonnegative measurable functions $f$ on $\Omega_{0}^{*}$

$$
\int Q(d \omega) \frac{f\left(\omega-t_{i}\right)}{N(0)}=\int Q(d \omega) \frac{f(\omega)}{N(0)}, \quad-\infty<i<\infty .
$$

We topologize $Q$ so that $Q_{n} \rightarrow Q$ if and only if $Q_{n}$ converges to $Q$ weakly and $\beta\left(Q_{n}\right) \rightarrow \beta(Q)$ as $n \rightarrow \infty$.

Theorem 7.6. The map from $\mathcal{P}$ to $\mathcal{Q}$ that takes $P$ to the corresponding tagged particle distribution $Q$ is a bomeomorphism of $\mathcal{P}$ onto 2 . This map is given explicitly by (7.11) and its inverse is given explicitly by (7.10) with $a(P)=1 / \beta(Q)$. 
Proof. The main thing that needs to be shown is that every $Q \in \mathcal{Q}$ arises as a tagged particle distribution. To this end, choose $Q \in 2$. Let $P$ be the probability measure on $\Omega^{*}$ defined by

$$
\int P(d \omega) f(\omega)=\frac{1}{\beta(Q)} \int Q(d \omega) \frac{1}{N(0)} \int_{0}^{\eta} 0 f(\omega-t) d t .
$$

Using the definition of 2 and the observation that $\eta_{0}\left(\omega-t_{i}\right)=t_{i+1}-t_{i}$, we conclude that

$$
\begin{aligned}
\int P(d \omega) f(\omega) & =\frac{1}{\beta(Q)} \int Q(d \omega) \frac{1}{N(0)} \int_{0}^{\eta} 0^{\left(\omega-t_{i}\right)} f\left(\omega-t_{i}-t\right) d t \\
& =\frac{1}{\beta(Q)} \int Q(d \omega) \frac{1}{N(0)} \int_{0}^{t_{i+1}{ }^{-t} i} f\left(\omega-t_{i}-t\right) d t \\
& =\frac{1}{\beta(Q)} \int Q(d \omega) \frac{1}{N(0)} \int_{t_{i}}^{t_{i+1}} f(\omega-t) d t
\end{aligned}
$$

By summing on $i$ we find that

$$
\int P(d \omega) f(\omega)=\frac{1}{i \beta(Q)} \int Q(d \omega) \frac{1}{N(0)} \int_{0}^{t} i f(\omega-t) d t
$$

Similarly

$$
\int P(d \omega) f(\omega-a)=\frac{1}{i \beta(Q)} \int Q(d \omega) \frac{1}{N(0)} \int_{a}^{a+t} i f(\omega-t) d t
$$

and hence

$$
\left|\int P(d \omega) f(\omega-a)-\int P(d \omega) f(\omega)\right| \leq \frac{2|a|}{i \beta(Q)}\|f\|_{\infty} \cdot
$$

Since $i$ can be made arbitrarily large we see that for $f$ bounded $\int P(d \omega) f(\omega-a)=$ $\int P(d \omega) f(\omega)$. This shows that $P$ is stationary.

Observe that

$$
\begin{array}{rlrl}
t_{i}(\omega-t) & =t_{i}(\omega)-t, & & 0 \leq t<t_{1}(\omega), \\
N\left(t_{i}(\omega-t), \omega-t\right)=N\left(0, \omega-t_{i}\right), & & 0 \leq t<t_{1}(\omega), \\
t_{i}(\omega)=-t_{-i}\left(\omega-t_{i}\right), & \omega \in \Omega_{0}^{*},
\end{array}
$$

and

$$
t_{i}(\omega)-t_{1}(\omega)=-t_{1-i}\left(\omega-t_{i}\right) .
$$

Thus by (7.21), for $f \geq 0$, 


$$
\begin{aligned}
B(Q) \int P(d \omega) f\left(\omega-t_{i}\right) N\left(t_{i}, \omega\right) 1_{A}\left(t_{i}\right) \\
=\int Q(d \omega) \frac{1}{N(0)} \int_{0}^{t_{1}(\omega)} f\left(\omega-t-t_{i}(\omega-t)\right) N\left(t_{i}(\omega-t), \omega-t\right) 1_{A}\left(t_{i}(\omega-t)\right) d t \\
=\int Q(d \omega) \frac{1}{N(0)} f\left(\omega-t_{i}\right) N\left(0, \omega-t_{i}\right) \int_{t_{i}-t_{1}{ }_{i}{ }^{t}(s) d s} \\
=\int Q(d \omega) \frac{1}{N(0)} f\left(\omega-t_{i}\right) N\left(0, \omega-t_{i}\right) \int_{-t_{1-i}\left(\omega-t_{i}\right)}^{-t_{A}(\omega) d s}(s) \\
=\int Q(d \omega) f(\omega) \int_{-t_{1}-i}^{-t_{-i}} 1_{A}(s) d s .
\end{aligned}
$$

By summing on $i$ we conclude that

$$
\int P(d \omega) \sum_{i} f\left(\omega-x_{i}\right) 1_{A}\left(x_{i}\right)=\frac{|A|}{\beta(Q)} \int Q(d \omega) f(\omega) .
$$

Taking $f=1$ in (7.22) we see that

$$
\int P(d \omega)_{i} N(A)=\frac{|A|}{\beta(Q)} .
$$

From (7.23) and the fact that $P$ is stationary we see that $P \in \mathcal{P}$. It follows from (7.22) that $Q$ is the tagged particle distribution corresponding to $P$.

We have now shown that the map described in the statement of Theorem 7.6 is an onto map. It follows from (7.10) that the map is one-to-one. Finally, we conclude from Theorem 5.1 that both the map and its inverse are continuous.

Let $\mathcal{Q}_{0}$ denote the collection of all stationary probability measures $Q_{0}$ on $\Xi$ such that $\gamma\left(Q_{0}\right)=\int Q_{0}(d \xi) \eta_{0}(\xi)<\infty$.

Let $Q$ be in 2. Then there is a unique $P \in \mathcal{P}$ having $Q$ as its tagged particle distribution. Let $Q_{0}$ be the spacing distribution corresponding to $P$. By Theorem 7.2 and Corollary 7.1, $Q_{0} \in \mathscr{Q}_{0}$. We refer to $Q_{0}$ as the spacing distribution corresponding to $Q$.

Theorem 7.7. The map from 2 to $2_{0}$ that takes $Q$ to the corresponding spacing distribution $Q_{0}$ is one-to-one and onto. It is given explicityly by (7.12) and its inverse is given explicitly by (7.13).

Proof. As in the proof of the previous theorem, the ma in difficulty is to prove that the map is onto. To this end, choose $Q_{0} \in \mathcal{Q}_{0}$. Let $Q$ be the probability measure on $\Omega_{0}^{*}$ defined by

$$
\int Q(d \omega) f(\omega)=\int Q_{0}(d \xi) f(\omega(\xi))
$$


Let $g$ be a nonnegative measurable function on $\Xi$. We will show that $(7.12)$ holds. As a first step we apply (7.24) to the function

obtaining

$$
f(\omega)=\frac{1}{N(0)} \sum_{i=0}^{N(0)-1} g\left(S^{-i} \xi(\omega)\right)
$$

$$
\int Q(d \omega) \frac{1}{N(0)} \sum_{i=0}^{N(0)-1} g\left(S^{-i} \xi(\omega)\right)=\int Q_{0}(d \xi) \frac{1}{N(0, \omega(\xi))} \sum_{i=0}^{N(0, \omega(\xi))-1} g\left(S^{-i} \xi(\omega(\xi))\right) .
$$

Thus to show that $(7.12)$ holds, we must verify

$$
\int Q_{0}(d \xi) \frac{1}{N(0, \omega(\xi))} \sum_{i=0}^{N(0, \omega(\xi))-1} g\left(S^{-i} \xi(\omega(\xi))\right)=\int Q_{0}(d \xi) g(\xi) .
$$

Set $\tau=\tau(\xi)=\min \left\{i \geq 0: \eta_{i}>0\right\}$. Then $\xi(\omega(\xi))=S^{\tau} \xi$ and hence $S^{-i} \xi(\omega(\xi))=$ $S^{\tau-i} \xi$. Consequently

$$
\begin{aligned}
& \int Q_{0}(d \xi) \frac{1}{N(0, \omega(\xi))} \sum_{i=0}^{N(0, \omega(\xi))-1} g\left(S^{-i} \xi(\omega(\xi))\right) \\
& =\int Q_{0}(d \xi) \frac{1}{N(0, \omega(\xi))} \sum_{i=0}^{N(0, \omega(\xi))-1} g\left(S^{\tau-i} \xi\right) \\
& \left.=\sum_{n=1}^{\infty} \sum_{m=0}^{n-1} \sum_{i=0}^{n-1} \frac{1}{n} \int Q_{0}(d \xi) g\left(S^{m-1} \xi\right)_{\{\tau=m, N(0, \omega(\xi))=n}\right\}(\xi) \\
& =\sum_{n=1}^{\infty} \sum_{m=0}^{n-1} \sum_{i=0}^{n-1} \frac{1}{n} \int Q_{0}(d \xi) g\left(S^{m-i} \xi\right)_{\left\{\eta_{m}>0, \eta_{m-n}>0, \eta_{m-1}=\cdots=\eta_{m-n+1}=0\right\}}(\xi) \\
& \left.=\sum_{n=1}^{\infty} \sum_{m=0}^{n-1} \sum_{i=0}^{n-1} \frac{1}{n} \int Q_{0}(d \xi) g(\xi) 1_{\left\{\eta_{i}>0, \eta_{i-n}>0, \eta_{i-1}\right.}=\cdots=\eta_{i-n+1}=0\right\}(\xi) \\
& =\sum_{n=1}^{\infty} \sum_{i=0}^{n-1} \int Q_{0}(d \xi) g(\xi) 1_{\{\tau=i, N(0, \omega(\xi))=n\}}(\xi)=\int Q_{0}(d \xi) g(\xi)
\end{aligned}
$$

which proves (7.25) and hence (7.12).

By setting $g(\xi)=\eta_{0}(\xi)$ in $(7.12)$ we conclude that

$$
\beta(Q)=\int Q(d \omega) \frac{\eta_{0}}{N(0)}=\int Q_{0}(d \xi) \eta_{0}(\xi)<\infty .
$$

To show that $Q \in \mathcal{Q}$ we need to show that for $f$ bounded 


$$
\int Q(d \omega) \frac{f(\omega-t-1)}{N(0)}=\int Q(d \omega) \frac{f(\omega)}{N(0)} .
$$

It follows from (7.12) that for $g$ bounded

$$
\begin{aligned}
0 & =\int Q_{0}(d \xi)\left[g(\xi)-g\left(S^{-1} \xi\right)\right]=\int Q(d \omega) \frac{1}{N(0)}\left[g(\xi(\omega))-g\left(S^{-N(0)} \xi(\omega)\right)\right] \\
& =\int Q(d \omega) \frac{1}{N(0)}\left[g(\xi(\omega))-g\left(\xi\left(\omega-t_{-1}\right)\right)\right] .
\end{aligned}
$$

Now let $g(\xi)=f(\omega(\xi))$. Then, for $\omega \in \Omega_{0}^{*}, g(\xi(\omega))=f(\omega(\xi(\omega)))=f(\omega)$ and $g\left(\xi\left(\omega-t_{-1}\right)\right)=f\left(\omega\left(\xi\left(\omega-t_{-1}\right)\right)\right)=f\left(\omega-t_{-1}\right)$. Consequently (7.27) holds and hence $Q \in 2$. It follows from (7.12) that $Q_{0}$ is the spacing distribution corresponding to $Q$. We have now shown that the map described in the statement of Theorem 7.7 is onto. It follows from (7.13) that the map is one-to-one.

We can make $\Xi$ into a complete separable metric space such that $\xi_{n} \rightarrow \xi$ if and only if $\omega\left(S^{i} \xi_{n}\right) \rightarrow \omega\left(S^{i} \xi\right)$ for all integers $i$. If the set $M$ of marks consists of a single point, then $\xi_{n} \rightarrow \xi$ if and only if $\eta_{i}\left(\xi_{n}\right) \rightarrow \eta_{i}(\xi)$ for all integers $i$.

We can make $2_{0}$ into a complete separable metric space such that $Q_{0}^{(n)} \rightarrow$ $Q_{0}$ if and only if $Q_{0}^{(n)}$ converges weakly to $Q_{0}$ and $\gamma\left(Q_{0}^{(n)}\right) \rightarrow \gamma\left(Q_{0}\right)$ as $n \rightarrow \infty$.

Theorem 7.8. The map from $\mathcal{P}$ to $\mathcal{Q}_{0}$ that takes $P$ to the corresponding spacing distribution $Q_{0}$ is a bomeomorphism of $\mathcal{P}$ onto $\mathcal{Q}_{0}$. This map is given explicitly by (7.14) and its inverse is given explicitly by (7.15) with $\alpha(P)=$ $1 / \gamma\left(Q_{0}\right)$.

Proof. Consider $P \in \mathcal{P}$. The maps $\omega \rightarrow x_{i}(\omega)$ and $\omega \rightarrow \xi(\omega)$ are continuous with probability one with respect to $P$. For suppose $\omega_{n}$ and $\omega_{0}$ are in $\Omega^{*}$ and $\omega_{n} \rightarrow \omega_{0}$. If either $X=Z$ or $N\left(0, \omega_{0}\right)=0$, then $x_{i}\left(\omega_{n}\right)^{n} \rightarrow x_{i}\left(\omega_{0}\right)$ for all $i$. Since $\omega\left(S^{i} \xi\left(\omega_{n}\right)\right)=\omega_{n}-x_{i}\left(\omega_{n}\right)$ we see that $\omega\left(S^{i} \xi\left(\omega_{n}\right)\right) \rightarrow \omega\left(S^{i} \xi\left(\omega_{n}\right)\right)$ for all $i$ and hence $\xi\left(\omega_{n}\right) \rightarrow \xi\left(\omega_{0}\right)$. But if $X \neq Z$, then $X=R$ and $P\left(N\left(0, \omega_{0}\right)=0\right)=1$.

In order to prove Theorem 7.8 we need only show that the map and its inverse are continuous. Suppose first that $P^{(n)}$ and $P$ are in $\mathscr{P}$ and $P^{(n)} \rightarrow P$. Let $A$ be a compact subset of $X$ such that $|A|=1$ and $|\partial \Lambda|=0$. Then

$$
\int P^{(n)}(d \omega) N(\Lambda)=\alpha\left(P^{(n)}\right) \rightarrow \alpha(P)=\int P(d \omega) N(A) .
$$

Since the distribution of $N(\Lambda)$ under $P^{(n)}$ converges to that under $P$, we conclude that

$$
\lim _{r \rightarrow \infty} \limsup _{n \rightarrow \infty} \int P^{(n)}(d \omega) N(A) 1_{\{N(A)>r\}}=0
$$


Let $g$ be a bounded continuous function on $\Xi$. By (7.28) and Theorem 7.4,

$$
\begin{aligned}
\int Q_{0}^{(n)}(d \xi) g(\xi) & =\frac{1}{\alpha\left(P^{(n)}\right)} \int P^{(n)}(d \omega) \sum_{i} 1_{A}\left(x_{i}\right) g\left(S^{-i} \xi(\omega)\right) \\
& \rightarrow \frac{1}{\alpha(P)} \int P(d \omega) \sum_{i} 1_{A}\left(x_{i}\right) g\left(S^{-i} \xi(\omega)\right)=\int Q_{0}(d \xi) g(\xi)
\end{aligned}
$$

and hence $Q_{0}^{(n)}$ converges weakly to $Q_{0}$. Since $\gamma\left(Q_{0}^{(n)}\right)=1 / \alpha\left(P^{(n)}\right) \rightarrow 1 / \alpha(P)=$ $\gamma\left(Q_{0}\right)$ we see that $Q_{0}^{(n)} \rightarrow Q_{0}$. Thus the map is continuous.

The proof that the inverse map is continuous follows by a similar argument based on (7.15).

Corollary 7.2. The map from 2 to $2_{0}$ that takes $Q$ to the corresponding spacing distribution $Q_{0}$ is a bomeomorphism from 2 onto $2_{0}$.

8. Random motion of rods. We will now apply the results of the previous section to study the motion of "rods" on the real line. By. a rod we mean a unit interval. By the position of a rod we mean the coordinate of its center. The motion will be such that no two rods have an interior point in common at any time. In the simplest setting, two rods interchange velocities when they collide.

We first motivate out general definition of the motion by working out the details of a simple numerical example. Consider three rods labeled 0,1 , and 2, starting out in the respective initial positions $-1,2$, and 7 and having the respective initial velocities $1,-1$, and -2 . If these rods could move independently of each other with these velocities, the position $X_{i}(t)$ of the $i$ th rod at time $t$ would be given by

$$
\begin{array}{ll}
X_{0}(t)=-1+t, & 0 \leq t<\infty, \\
X_{1}(t)=2-t, & 0 \leq t<\infty, \\
X_{2}(t)=7-2 t, & 0 \leq t<\infty .
\end{array}
$$

Our rods, however, are constrained not to overlap. We let this be done by supposing that two rods interchange velocities when they collide. The motion is now as illustrated in Figure 1.

Let $Y_{i}(t)$ be the true position of the $i$ th rod at time $t$. We see by inspection of Figure 1 that

$$
\begin{array}{rlrlrl}
Y_{0}(t)=-1+t, & 0 \leq t<1, & Y_{1}(t)=2-t, & 0 \leq t<1, \\
=1-t, & 1 \leq t<4, & & =t, & 1 \leq t<2, \\
=5-2 t, & 4 \leq t ; & & =6-2 t, & 2 \leq t<4, \\
& =2-t, & 4 \leq t ; \\
Y_{2}(t)=7-2 t, & 0 \leq t<2, & \\
& =1+t, & 2 \leq t . &
\end{array}
$$




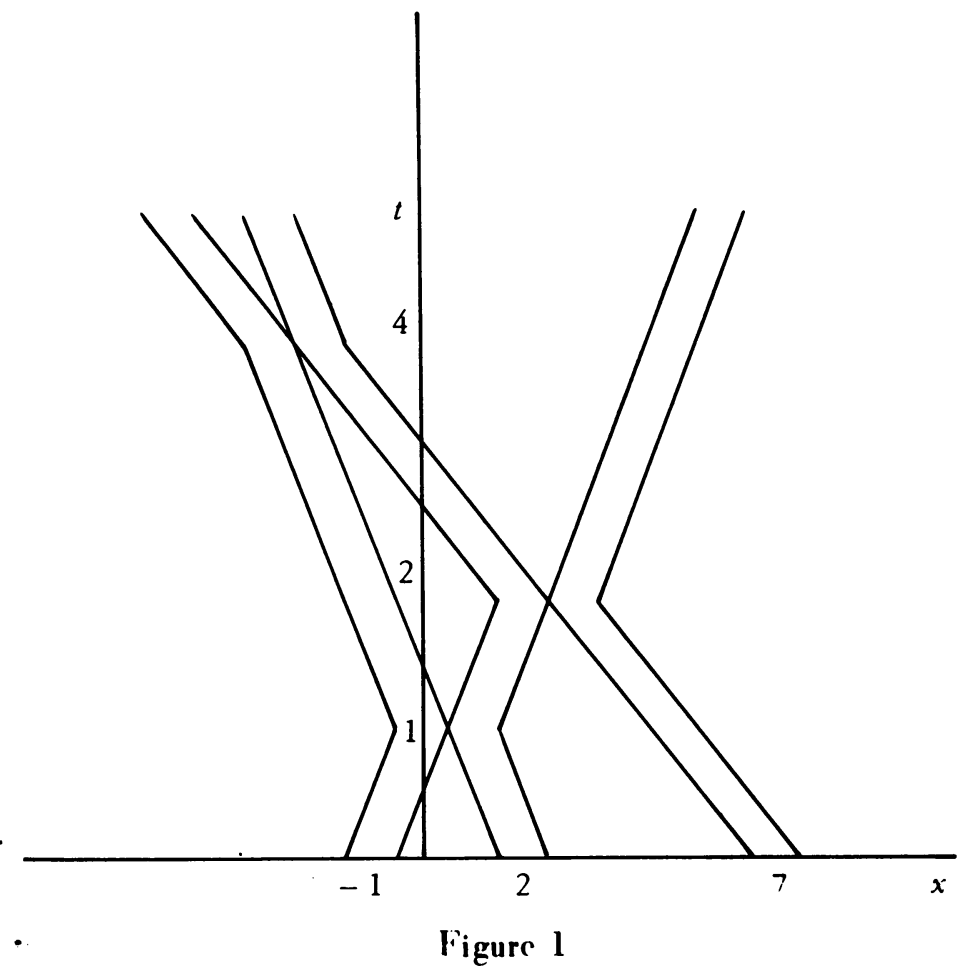

For each $t$ the functions $Y_{i}(t)$ can be obtained by first writing $X_{i}(t)-i$ in nondecreasing order and then adding $i$ to the resulting values. For example, if $t \geq 4$ we obtain

$$
X_{2}(t)-2=5-2 t, \quad X_{1}(t)-1=1-t, \quad X_{0}(t)-0=-1+t
$$

By adding $i$ to the $i$ th such value we find that

$$
Y_{0}(t)=5-2 . t, \quad Y_{1}(t)=2-t, \quad Y_{2}(t)=1+t,
$$

which agrees with our previous formulas for $t \geq 4$.

Consider now a doubly infinite sequence of motions $X_{i}(t), 0 \leq t<\infty$, satisfying the conditions

$$
X_{i+1}(0) \geq X_{i}(0)+1, \quad-\infty<i<\infty
$$

and

$$
\lim _{i \rightarrow \pm \infty} X_{i}(t)-i= \pm \infty, \quad 0 \leq t<\infty
$$

For positive integers $m$ and $n$ let $Y_{i m n}(t)-i,-m \leq i \leq n$, be the numbers $X_{i}(t)-i,-m \leq i \leq n$, arranged in nondecreasing order. It follows from (8.2) that, 
for fixed $i$ and $t, Y_{i m n}(t)$ is constant in $m$ and $n$ for $m$ and $n$ sufficiently large. We denote the constant value by $Y_{i}(t)=\lim _{m, n \rightarrow \infty} Y_{i m n}(t)$. Clearly

$$
Y_{i}(t)-i \leq Y_{i}(t)-j, \quad i \leq j \text {. }
$$

It follows from (8.1) that

$$
Y_{i}(0)=X_{i}(0), \quad-\infty<i<\infty .
$$

Similarly for each pair of positive integers $m$ and $n$ and each $t \geq 0$ there is a oneto-one map $i \rightarrow \nu_{i m n}(t)$ of $\{i:-m \leq i \leq n\}$ onto itself such that $\nu_{i m n}(t)<\nu_{j m n}(t)$ if and only if either

$$
X_{i}(t)-i<X_{i}(t)-j
$$

or

$$
X_{i}(t)-i=X_{j}(t)-j \quad \text { and } i<j .
$$

The function $\nu_{i}(t)=\lim _{m, n \rightarrow \infty} \nu_{i m n}(t)$ exists and is a one-to-one function of the integers onto itself such that $\nu_{i}(t)<\nu_{j}(t)$ if and only if (8.5) or (8.6) holds. Moreover, $\nu_{i}(0)=i$ and

$$
Y_{\nu_{i}(t)}(t)-\nu_{i}(t)=X_{i}(t)-i, \quad 0 \leq t<\infty
$$

This construction was suggested by that of Harris [3].

Suppose we replace the functions $X_{i}(t)$ by

$$
X_{i}^{*}(t)=X_{i+i_{0}}(t)+c .
$$

Then

and

$$
\begin{aligned}
Y_{i}^{*}(t) & =Y_{i+i_{0}}(t)+c, \\
\nu_{i}^{*}(t) & =\nu_{i+i_{0}}(t)-i_{0}, \\
Y_{\nu_{i}^{*}(t)}^{*}(t)-\nu_{i}^{*}(t) & =X_{i}^{*}(t)-i,
\end{aligned}
$$

$$
Y_{\nu_{i}^{*}(t)}^{*}(t)=Y_{\nu_{i+i_{0}}(t)}(t)+c .
$$

Let $\left\{\xi_{i}(t), t \geq 0\right\}_{-\infty}^{\infty}$ be independent and identically distributed stochastic processes such that $\xi_{i}(0)=0$ and

$$
E \sup _{0 \leq t \leq T}\left|\xi_{i}(t)\right|<\infty, \quad 0 \leq T<\infty .
$$


Let $\left\{X_{i}(0)\right\}_{-\infty}^{\infty}$ be random variables which collectively are independent of $\left\{\xi_{i}(t), t \geq 0\right\}_{-\infty}^{\infty}$ and such that $X_{i+1}(0) \geq X_{i}(0)+1, X_{0}(0) \leq 0<X_{1}(0)$, and

$$
\liminf _{i \rightarrow \pm \infty} \frac{X_{i}(0)}{i}>1
$$

each with probability one. Set $X_{i}(t)=X_{i}(0)+\xi_{i}(t), t \geq 0$. Then with probability one (8.2) holds for all $t \geq 0$, so $\left\{Y_{i}(t), t \geq 0\right\}$ is well defined. We can think of $\left\{X_{i}(t)\right\}$ as a random counting measure $N(t)$ defined by $\int f(x) N(t)(d x)=\Sigma_{i} f\left(X_{i}(t)\right)$. Similarly we can think of $\left\{Y_{i}(t)\right\}$ as a random counting measure. Let $P(t)$ denote the distribution of $\left\{Y_{i}(t)\right\}$ and let $\bar{P}(t)$ denote the distribution of $\left\{X_{i}(t)\right\}$. Then $P(0)=\bar{P}(0)$.

Suppose now that $\bar{P}(0)$ is a stationary distribution. It follows from (8.14) that $0<a(\bar{P}(0))<1$. For any fixed $t \geq 0,\left\{\left(X_{i}(0), X_{i}(t)\right)\right\}$ defines a translation invariant marked motion process. Thus $\bar{P}(t)$ is stationary and $\alpha(\bar{P}(t))=\alpha(\bar{P}(0))$. It follows from (8.9) that $\left\{\left(Y_{i}(0), Y_{i}(t)\right)\right\}$ is a translation invariant marked motion process. Thus $P(t)$ is stationary and $\alpha(P(t))=\alpha(P(0))=\alpha(\bar{P}(0))$. It follows from (8.12) that $\left\{\left(Y_{\nu_{i}(0)}(0), Y_{\nu_{i}(t)}(t)\right)\right\}$ is a translation invariant marked motion process. Since $\nu_{i}(t)$ is a one-to-one map of the integers onto itself $\left\{Y_{\nu_{i}(t)}(t)\right\}=\left\{Y_{i}(t)\right\}$ in the sense that they both define the same counting measure. Thus $\left\{Y_{\nu_{i}(t)}(t)\right\}$ also has $P(t)$ for its distribution.

Let $Q(t)$ and $Q_{0}(t)$ denote the tagged particle distribution and spacing distribution corresponding to $P(t)$. Let $\left\{\eta_{i}\right\}$ be distributed according to $Q_{0}(t)$. Then $\left\{\eta_{i}-1\right\}$ is a spacing process, whose distribution we denote by $Q_{0}^{-1}(t)$. Let $Q^{-1}(t)$ and $P^{-1}(t)$ denote the distributions in $\mathcal{Q}$ and $\mathcal{P}$ corresponding to $Q_{0}^{-1}(t)$.

Let $\bar{Q}(0)$ and $\bar{Q}_{0}(0)$ denote the tagged particle distribution and spacing distribution corresponding to $\bar{P}(0)$. Let $\left\{\eta_{i}\right\}$ be distributed according to $\bar{Q}_{0}(0)$. Then, for $c \leq 1,\left\{\eta_{i}-c\right\}$ is a spacing process, whose distribution we denote by $\bar{Q}_{0}^{-c}(0)$. Let $\bar{Q}^{-c}(0)$ and $\bar{P}^{-c}(0)$ denote the distributions in $\mathscr{Q}$ and $\mathcal{P}$ corresponding to $\bar{Q}_{0}^{-c}(0)$. Consider particles placed down according to the distribution $\bar{P}^{-c}(0)$ and translated by the independent motions $\left\{\xi_{i}(t), t \geq 0\right\}$. Let $\bar{P}^{-c}(t)$ be the distribution of the particles at time $t$. Let $\bar{Q}^{-c}(t)$ and $\bar{Q}_{0}^{-c}(t)$ be the tagged particle distribution and spacing distribution corresponding to $\bar{P}^{-c}(t)$.

The next theorem enables us to reduce results involving the interacting system $\left\{Y_{i}(t), t \geq 0\right\}$ to those of the independent motions $\left\{X_{i}(t), t \geq 0\right\}$.

Theorem 8.1. $Q^{-1}(t)=\bar{Q}^{-1}(t), t \geq 0$.

Proof. From (8.7) we see that

$$
Y_{\nu_{i}(t)}(t)-Y_{\nu_{0}(t)}(t)-\left(\nu_{i}(t)-\nu_{0}(t)\right)=X_{i}(t)-i-X_{0}(t), \quad t \geq 0 .
$$


Suppose that $\left\{X_{i}(0)\right\}=\left\{Y_{i}(0)\right\}=\left\{Y_{\nu_{i}(0)}(0)\right\}$ is distributed according to $Q(0)$. Choose $t>0$. It follows from Theorem 6.5 that $\left\{Y_{\nu_{i}(t)}(t)-Y_{\nu_{0}(t)}(t)\right\}$ is distributed according to $Q(t)$. But $\nu_{i}(t)-\nu_{0}(t)$ is the rank of $Y_{\nu_{i}(t)}(t)$ in $\left\{Y_{\nu_{i}(t)}(t)\right\}$, taking the rank of $Y_{\nu_{0}(t)}(t)$ to be zero. By looking at the corresponding spacing distributions we see that

$$
\left\{Y_{\nu_{i}(t)}(t)-Y_{\nu_{0}(t)}(t)-\left(\nu_{i}(t)-\nu_{0}(t)\right)\right\}
$$

is distributed according to $Q^{-1}(t)$.

Choose $0 \leq c<1$. Then $\left\{X_{i}(0)-c i\right\}$ is distributed according to $\bar{Q}^{-c}(0)$. Moreover, the points $\left\{X_{i}(0)-c i\right\}$ are distinct with probability one. We conclude from Theorem 6.5 that $\left\{X_{. i}(t)-c i-X_{0}(t)\right\}$ is distributed according to $\bar{Q}^{-c}(t)$. Clearly $\bar{Q}_{0}^{-c}(0)$ converges to $\bar{Q}_{0}^{-1}(0)$ as $c \rightarrow 1$. It follows from Theorem 7.8 that $\bar{P}^{-c}(0)$ converges to $\bar{P}^{-1}(0)$ as $c \rightarrow 1$. We conclude from Corollary 6.1 that $\bar{P}^{-c}(t)$ converges to $\bar{P}^{-1}(t)$ as $c \rightarrow 1$. Thus by Theorem $7.6, \bar{Q}^{-c}(t) \rightarrow \bar{Q}^{-1}(t)$ as $c \rightarrow 1$. It is obvious that the distribution of $\left\{X_{i}(t)-c i-X_{.0}(t)\right\}$ converges weakly to that of $\left\{X_{i}(t)-i-X_{0}(t)\right\}$ as $c \rightarrow 1$. Consequently the latter is distributed according to $\bar{Q}^{-1}(t)$. The theorem now follows from (8.15).

Let $P_{\lambda}$ denote the distribution of the Poisson process with parameter $\lambda$ on $R$. Let $Q_{\lambda}$ and $\left(Q_{0}\right)_{\lambda}$ denote the corresponding tagged particle distribution and spacing distribution. Let $\left\{\eta_{i}\right\}$ be distributed according to $\left(Q_{0}\right)_{\lambda}$. Then $\left\{\eta_{i}\right\}$ is a collection of independent random variables, each having an exponential distribution with parameter $\lambda$. Let $\left(Q_{0}\right)_{\lambda}^{1}$ be the distribution of $\left\{\eta_{i}+1\right\}$ and let $Q_{\lambda}^{1}$ and $P_{\lambda}^{1}$ be the corresponding distributions in $\mathcal{2}$ and $\mathcal{P}$. The next theorem shows that $P_{\lambda}^{1}$ is an invariant distribution for the $\left\{Y_{i}(t)\right\}$ process.

Theorem 8.2. If $P(0)=P_{\lambda}^{1}$, then $P(t)=P_{\lambda}^{1}$ for all $t \geq 0$.

Proof. Suppose $P(0)=P_{\lambda}^{1}$. Then $\bar{P}(0)=P(0)=P_{\lambda}^{1}$, so $\bar{Q}_{0}(0)=\left(Q_{0}\right)_{\lambda}^{1}$. Thus $\bar{Q}_{0}^{-1}(0)=\left(Q_{0}\right)_{\lambda}$ and hence $\bar{P}^{-1}(0)=P_{\lambda}$. The Poisson distribution is well known to be invariant for motions defined by independent translations. Thus $\bar{P}^{-1}(t)=$ $P_{\lambda}$ and hence $\bar{Q}^{-1}(t)=Q_{\lambda}$. We conclude from Theorem 8.1 that $Q^{-1}(t)=Q_{\lambda}$. Thus $Q_{0}^{-1}(t)=\left(Q_{0}\right)_{\lambda}$ and hence $Q_{0}(t)=\left(Q_{0}\right)_{\lambda}^{1}$. Consequently $P(t)=P_{\lambda}^{1}$ as desired.

In order to obtain convergence to the invariant distribution, we need to assume that

$$
\begin{aligned}
\lim _{t \rightarrow \infty} \sum_{n=-\infty}^{\infty}\left|P\left(a n \leq \xi_{0}(t)<a(n+1)\right)-P\left(a(n-1) \leq \xi_{0}(t)<a_{n}\right)\right|=0, & \\
& 0<a<\infty .
\end{aligned}
$$

This condition is satisfied if $\xi_{0}(t)=v_{0} t, 0 \leq t<\infty$, where $v_{0}$ is a positive random variable having an absolutely continuous distribution, or if $\xi_{0}(t), 0 \leq t<\infty$, 
is a nondegenerate process with stationary independent increments (see Stone [18]). It is also satisfied if $\xi_{0}(t), t \geq 0$, is normally distributed with a variance approaching infinity as $t \rightarrow \infty$.

Recall that $\bar{N}$ denotes the limit of $N([-T, T]) / 2 T$ as $T \rightarrow \infty$. We will consider distributions $P \in \mathcal{P}$ such that

$$
\bar{N}=\alpha(P) \quad \text { with probability } 1 \text { w.r.t. } P \text {. }
$$

In the next theorem we will see that under appropriate conditions the distribution of $\left\{Y_{i}(t)\right\}$ converges to an invariant distribution as $t \rightarrow \infty$.

Theorem 8.3. Suppose that (8.16) bolds and that $\left\{Y_{i}(0)\right\}$ is distributed according to a $P(0) \in \mathscr{P}$ that satisfies (8.17). Then

$$
\lim _{t \rightarrow \infty} P(t)=P_{\lambda}^{1}
$$

where $\lambda=\alpha(P(0)) /(1-\alpha(P(0)))$.

Proof. Suppose thạt $P(0) \in \mathcal{P}$ satisfies (8.17). It follows from Theorem 7.4 that

$$
\bar{\eta}=\lim _{i \rightarrow \infty} \frac{\eta_{0}+\cdots+\eta_{i-1}}{i}=\frac{1}{\alpha(P(0))}
$$

with probability one w.r.t. $Q_{0}(0)$. From this we conclude that

$$
\bar{N}=\frac{a(P(0))}{1-a(\overline{P(0)})} \quad \text { with probability } 1 \text { w.r.t. } \bar{P}^{-1}(0) .
$$

From (8.16), (8.19), Theorem 2 of Stone [8], and Theorem 2.1 of Harris [3] it follows that $\lim _{t \rightarrow \infty} \bar{P}^{-1}(t)=P_{\lambda}$, where $\lambda=\alpha(P(0)) /(1-\alpha(P(0)))$. Thus by Theorem $7.6 \lim _{t \rightarrow \infty} \bar{Q}^{-1}(t)=Q_{\lambda}$. By Theorem $8.1 \lim _{t \rightarrow \infty} Q^{-1}(t)=Q_{\lambda}$. It follows from Corollary 7.2 that $\lim _{t \rightarrow \infty} Q_{0}^{-1}(t)=\left(Q_{0}\right)_{\lambda}$. Consequently $\lim _{t \rightarrow \infty} Q_{0}(t)=$ $\left(Q_{0}\right)_{\lambda}^{1}$. Finally, we conclude from Theorem 7.8 that $(8.18)$ holds as desired.

\section{REFERENCES}

1. N. Dunford and J. T. Schwartz, Linear operators. I: General theory, Pure and Appl. Math., vol. 7, Interscience, New York, 1958. MR 22 \#8302.

2. T. E. Harris, Diffusion with "collisions" between particles, J. Appl. Probability 2 (1965), 323-338. MR $32 \# 1750$.

3. - Random measures and motions of point processes, Z. Wahrscheinlichkeitstheorie und Verw. Gebiete 18 (1971), 85-115.

4. J. Mecke, Stationäre Zufällige Masse auf lokalkompakten Abelschen Gruppen, Z. Wahrscheinlichkeitstheorie und Verw. Gebiete 9 (1967), 36-58. MR 37 \#3611. 
5. C. Ryll-Nardzewski, Remarks on processes of calls, Proc. Fourth Berkeley Sympos. Math. Statist. and Probability, vol. 2, Univ. of California Press, Berkeley, Calif., 1961, pp. 455-465. MR $25 \# 3575$.

6. I. M. Slivnjak, Some properties of stationary streams of homogeneous random events, Teor. Verojatnost. i Primenen. 7 (1962), 347-352 = Theor. Probability Appl. 7 (1962), 336-341. MR 27. \#832.

7. F. L. Spitzer, Interaction of Markov processes, Advances in Math. 5 (1970), 246290. MR 42 \#3856.

8. C. J. Stone, On a theorem of Dobrushin, Ann. Math. Statist. 39 (1968), 13911401. MR 37 \#6996.

DEPARTMENT OF MATHEMATICS, UNIVERSITY OF CALIFORNIA, LOS ANGELES, CALIFORNIA 90024 